\title{
Dichotomous keys to the species of Solanum L. (Solanaceae) in continental Africa, Madagascar (incl. the Indian Ocean islands), Macaronesia and the Cape Verde Islands
}

\author{
Sandra Knapp', Maria S. Vorontsova², Tiina Särkinen ${ }^{3}$ \\ I Department of Life Sciences, Natural History Museum, Cromwell Road, London SW7 5BD, UK 2 Compa- \\ rative Plant and Fungal Biology Department, Royal Botanic Gardens, Kew, Richmond, Surrey TW9 3AE, UK \\ 3 Royal Botanic Garden Edinburgh, 20A Inverleith Row, Edinburgh EH3 5LR, UK \\ Corresponding author: Sandra Knapp (s.knapp@nhm.ac.uk) \\ Academic editor: Leandro Giacomin | Received 9 March 2019 | Accepted 5 June 2019 | Published 19 July 2019 \\ Citation: Knapp S, Vorontsova MS, Särkinen T (2019) Dichotomous keys to the species of Solanum L. (Solanaceae) in \\ continental Africa, Madagascar (incl. the Indian Ocean islands), Macaronesia and the Cape Verde Islands. PhytoKeys 127: \\ 39-76. https://doi.org/10.3897/phytokeys.127.34326
}

\begin{abstract}
Solanum L. (Solanaceae) is one of the largest genera of angiosperms and presents difficulties in identification due to lack of regional keys to all groups. Here we provide keys to all 135 species of Solanum native and naturalised in Africa (as defined by World Geographical Scheme for Recording Plant Distributions): continental Africa, Madagascar (incl. the Indian Ocean islands of Mauritius, La Réunion, the Comoros and the Seychelles), Macaronesia and the Cape Verde Islands. Some of these have previously been published in the context of monographic works, but here we include all taxa. The paper is designed to be used in conjunction with the web resource Solanaceae Source (www.solanaceaesource.org) and hyperlinks provide access to online descriptions, synonymy and images (where available) of each species. All taxa treated and specimens seen are included in searchable Suppl. material 1, 2.
\end{abstract}

\section{Keywords}

Africa, Aldabra, Azores, Canary Islands, Cape Verde, Comoros, cultivated plants, identification, keys, Madagascar, Madeira, Mauritius, La Réunion, Seychelles, Solanum, weeds

Copyright Sandra Knapp et al. This is an open access article distributed under the terms of the Creative Commons Attribution License (CC BY 4.0), which permits unrestricted use, distribution, and reproduction in any medium, provided the original author and source are credited. 


\section{Introduction}

Solanum L. (Solanaceae) is one of the largest of angiosperm genera (Frodin 2004) with ca. 1,200 species distributed worldwide with species on all continents except Antarctica. The greatest species diversity in the genus occurs in the Neotropics (see Bohs 2005), but significant diversity also occurs in the Old World, with Africa and Australia particularly important areas for diversification (see Vorontsova and Knapp 2016; Echeverría-Londoño et al. 2018). Due to its large number of species and the number of introductions and cultivated taxa, Solanum is often an identification challenge for nonspecialists. Recent completion of several large monographic treatments of the Solanum of Africa (e.g. Vorontsova and Knapp 2016; Knapp and Vorontsova 2016; Särkinen et al. 2018) as part of the US National Science Foundation funded Planetary Biodiversity Inventory project "PBI Solanum" means we can now provide keys for the genus across the continent and for adjacent islands such as Madagascar and Macaronesia. Some of these have been published in the Open Access literature (e.g. Knapp 2013; Knapp and Vorontsova 2016; Särkinen et al. 2018), but the largest of these, treating the spiny solanums (Vorontsova and Knapp 2016) is not. Several species that are either naturalised (e.g. members of the Brevantherum Clade) or cultivated (tree tomatoes, pepinos, potatoes and tomatoes) in the region are also not treated as part of these monographic treatments, although complete species descriptions and photographs are provided on the web resource Solanaceae Source (www.solanaceaesource.org).

Here we provide dichotomous keys that include all groups and species (native, naturalised and widely cultivated; see Table 1 for species list) of Solanum occurring in continental Africa, Madagascar (incl. the Indian Ocean islands of Mauritius, La Réunion, the Comoros, and the Seychelles), Macaronesia and the Cape Verde Islands to facilitate identification across the region. Taxa occurring in each country in the region are shown in Table 2 and a map of Solanum diversity (all taxa) is presented in Figure 1. Keys to individual groups are also provided for the 135 Solanum species occurring in the region (see Table 1 and Appendix 1 for a species list). We hope that these keys will encourage collection and documentation of Solanum across Africa and uncover new distributions and perhaps new species for the region.

\section{Materials and methods}

We modified keys from published monographs for groups of Solanum from the botanical continent "Africa" as defined in the World Geographical Scheme for Recording Plant Distributions (WGSRPD; Brummitt 2001). This corresponds basically to the countries of the continent of Africa, but excludes the Sinai Peninsula (politically part of Egypt and in WGSRPD part of Western Asia). It also includes islands grouped as Macaronesia (Azores, Canary Islands, Madeira and the Cape Verde Islands) and Madagascar and other Indian Ocean islands east to Rodrigues. 
We assessed distribution using the published monographs, with additional data points added from subsequent herbarium visits. All specimens seen for these keys can be seen in the Supplemental File and in the dataset published on the NHM Data Portal (https://doi.org/10.5519/0042549). For descriptions of the taxa, users are referred to the original publications or the Solanaceae Source website (www.solanaceaesource. org), where all species treated here are described and synonymy listed.

To access descriptions on the Solanaceae Source website, begin by typing the species name in the search box in the upper right-hand part of the screen banner (tick the option "Taxonomy" below the box); when the correct name you are searching for appears, select it, then push the "Search" button to the right of the search box (if you do not push the "Search" button, nothing will happen). You will be taken to the species page, where images and synonyms appear on the opening page; to access descriptions, click on the "Description" tab where information can be obtained. Up-to-date specimen details are not currently available on the website but can be found as described above.

\section{Keys}

Solanum can be divided into 13 major clades or monophyletic groups (Bohs 2005; Weese and Bohs 2007; Särkinen et al. 2013; see Figures 2 and 3 for photographs illustrating representative morphology of these groups in Africa). The largest monophyletic clade is the Leptostemonum clade, or the "spiny solanums", which comprises approximately half of the species diversity of the genus; divisions within that clade have been defined by Stern et al. (2011), Vorontsova et al. (2013) and Aubriot et al. (2016). This group is rapidly diversifying in the Old World (Echeverría-Londoño et al. 2018), with most taxa occurring in the Old World belonging to a single monophyletic group. Previous treatments (e.g. Whalen 1984; Jaeger 1985; Jaeger and Hepper 1986) had suggested the African taxa were members of, or closely related to New World groups. More information on the phylogenetic relationships of African and Asian members of the Leptostemonum Clade can be found in Vorontsova et al. (2013) and Aubriot et al. (2016). Other clades with significant species diversity in Africa (as defined here) are the African non-spiny (ANS) and Normania Clades (both endemic to the region; see Bohs and Olmstead 2001) and the Morelloid Clade (with a number of widespread weedy taxa, see Särkinen et al. 2018). Other clades such as the Geminata, Brevantherum and Potato Clades are represented only by introduced or cultivated species. The Dulcamaroid Clade has a single species native to Mediterranean northern Africa and Macaronesia and two cultivated taxa that can become naturalised (Knapp 2013). In order to facilitate identification and to assist with the discovery of novelties from the region, we provide a key to the major groups (clades) of Solanum following the most recent phylogeny of the genus (Särkinen et al. 2013) and additional dichotomous keys to the species within each group. Groups are ordered as they occur as branches in the phylogeny of Särkinen et al. (2013). 
Table I. The 135 species of Solanum (native, naturalised and widely cultivated) occurring in Africa as defined by Brummitt (2001), with their places of original publication and clade membership as currently understood (Major Clades sensu Bohs 2005; minor clades are divisions within these sensu Bohs 2007; Stern et al. 2011; Vorontsova et al. 2103; Särkinen et al. 2015, 2018; Aubriot et al. 2016; Tepe et al. 2016).

\begin{tabular}{|c|c|c|c|}
\hline Species & Place of original publication & Major Clade & Minor clade \\
\hline Solanum aculeastrum Dunal & Prodr. [A. P. de Candolle] 13(1): 366. 1852. & Leptostemonum & Old World-Africa \\
\hline Solanum aculeatissimum Jacq. & Collectanea [Jacquin] 1: 100. 1787 ['1786']. & Leptostemonum & Acanthophora \\
\hline Solanum adoense Hochst. ex A.Rich. & Tent. Fl. Abyss. 2: 105. 1850 [1851]. & Leptostemonum & Old World-Africa \\
\hline Solanum aethiopicum $\mathrm{L}$. & Cent. Pl. 2: 10. 1756. & Leptostemonum & Old World-Africa \\
\hline Solanum africanum Mill. & Gard. Dict. ed. 8, no. 26. 1768. & $\begin{array}{l}\text { African non-spiny } \\
\text { (ANS) }\end{array}$ & \\
\hline Solanum agnewiorum Voronts. & Phytotaxa 10: 32. 2010. & Leptostemonum & Old World-Africa \\
\hline Solanum agrarium Sendtn. & $\begin{array}{l}\text { Fl. Bras. (Martius) 10: 68, fig. 5, 32-33. } \\
1846 .\end{array}$ & Leptostemonum & Gardneri \\
\hline Solanum aldabrense C.H.Wright & Kew Bull. 1894: 149. 1894. & Leptostemonum & Old World \\
\hline Solanum americanum Mill. & Gard. Dict. ed. 8, no. 5. 1768. & Morelloid & Black nightshade \\
\hline Solanum anguivi Lam. & Tabl. Encycl. 2: 23. 1794. & Leptostemonum & Old World-Africa \\
\hline Solanum anomalum Thonn. & Beskr. Guin. Pl. 1261827. & Leptostemonum & Old World-Africa \\
\hline Solanum arundo Mattei & $\begin{array}{l}\text { Boll. Reale Orto Bot. Giardino Colon. } \\
\text { Palermo 7: } 188.1908 .\end{array}$ & Leptostemonum & Old World-Africa \\
\hline Solanum atropurpureum Schrank & Syll. Ratisb. 1: 200. 1824. & Leptostemonum & Acanthophora \\
\hline Solanum aureitomentosum Bitter & Repert. Spec. Nov. Regni Veg. 11: 18. 1912. & Leptostemonum & Old World-Africa \\
\hline Solanum batoides D’Arcy \& Rakot. & Fl. Madag., Fam. 176: 75. 1994. & Leptostemonum & $\begin{array}{l}\text { Old World- } \\
\text { Madagascar }\end{array}$ \\
\hline Solanum betaceum Cav. & Anales Hist. Nat. 1: 44.1799. & Cyphomandra & Pachyphylla \\
\hline Solanum betroka D’Arcy \& Rakot. & Fl. Madag., Fam. 176: 77. 1994. & $\begin{array}{l}\text { African non-spiny } \\
\text { (ANS) }\end{array}$ & \\
\hline Solanum bumeliifolium Dunal & Prodr. [A. P. de Candolle] 13(1): 292. 1852. & Leptostemonum & $\begin{array}{l}\text { Old World- } \\
\text { Madagascar }\end{array}$ \\
\hline Solanum burchellii Dunal & Prodr. [A. P. de Candolle] 13(1): 291. 1852. & Leptostemonum & Old World-Africa \\
\hline $\begin{array}{l}\text { Solanum campylacanthum Hochst. } \\
\text { ex A.Rich. }\end{array}$ & Tent. Fl. Abyss. 2: 102. 1850. & Leptostemonum & Old World-Africa \\
\hline Solanum capense $\mathrm{L}$. & Syst. ed. 10: 935. 1759. & Leptostemonum & Old World-Africa \\
\hline Solanum capsicoides All. & $\begin{array}{l}\text { Auct. Syn. Meth. Stirp. Hort. Regii Taur. } \\
64.1773 .\end{array}$ & Leptostemonum & Acanthophora \\
\hline Solanum catombelense Peyr. & $\begin{array}{c}\text { Sitzungsber. Kaiserl. Akad. Wiss., Math.- } \\
\text { Naturwiss. Cl. 38: 576. } 1860 .\end{array}$ & Leptostemonum & Old World-Africa \\
\hline Solanum cerasiferum Dunal & Prodr. [A. P. de Candolle] 13(1): 365. 1852. & Leptostemonum & Old World-Africa \\
\hline Solanum chenopodioides Lam. & Tabl. Encycl. 2: 18. 1794. & Morelloid & Black nightshade \\
\hline Solanum chrysotrichum Schltdl. & Linnaea 19: 304. 1847. & Leptostemonum & Torva \\
\hline Solanum coagulans Forssk. & Fl. Aegypt.-Arab. 47. 1775. & Leptostemonum & Old World-Africa \\
\hline Solanum cordatum Forssk. & Fl. Aegypt.-Arab. 47. 1775. & Leptostemonum & [not assigned] \\
\hline $\begin{array}{l}\text { Solanum croatii D'Arcy \& } \\
\text { R.C.Keating }\end{array}$ & Phytologia 34: 282. 1976. & Leptostemonum & $\begin{array}{l}\text { Old World- } \\
\text { Madagascar }\end{array}$ \\
\hline Solanum cyaneopurpureum De Wild. & Pl. Bequaert. 1: 425. 1922. & Leptostemonum & Old World-Africa \\
\hline Solanum cymbalariifolium Chiov. & Boll. Soc. Bot. Ital. 1925: 107. 1925. & Leptostemonum & Old World-Africa \\
\hline $\begin{array}{l}\text { Solanum dasyphyllum } \\
\text { Schumach. \& Thonn. }\end{array}$ & Beskr. Guin. Pl. 126 [146]. 1827. & Leptostemonum & Old World-Africa \\
\hline Solanum dennekense Dammer & Bot. Jahrb. Syst. 38: 57. 1905. & Leptostemonum & Old World-Africa \\
\hline Solanum diphyllum $\mathrm{L}$. & Sp. Pl. 184. 1753. & Geminata & \\
\hline Solanum elaeagnifolium $\mathrm{Cav}$. & Icon. 3: 22, tab. 243. 1795. & Leptostemonum & Elaeagnifolium \\
\hline Solanum erianthum D.Don & Prodr. Fl. Nep. 96. 1825. & Brevantherum & \\
\hline Solanum erythracanthum Dunal & Prodr. [A. P. de Candolle] 13(1): 201. 1852. & Leptostemonum & $\begin{array}{l}\text { Old World- } \\
\text { Madagascar }\end{array}$ \\
\hline Solanum forskalii Dunal & Hist. Nat. Solanum 237. 1813. & Leptostemonum & Old World-Africa \\
\hline Solanum giganteum Jacq. & Collectanea [Jacquin] 4: 125. 1791. & Leptostemonum & Old World-Africa \\
\hline
\end{tabular}




\begin{tabular}{|c|c|c|c|}
\hline Species & Place of original publication & Major Clade & Minor clade \\
\hline Solanum glabratum Dunal & Hist. Nat. Solanum 240. 1813. & Leptostemonum & Old World-Africa \\
\hline Solanum goetzei Dammer & Bot. Jahrb. Syst. 28: 473. 1900. & Leptostemonum & Old World-Africa \\
\hline Solanum guineense $\mathrm{L}$. & Sp. Pl. 184. 1753. & $\begin{array}{l}\text { African non-spiny } \\
\text { (ANS) }\end{array}$ & \\
\hline $\begin{array}{l}\text { Solanum hastifolium } \\
\text { Hochst. ex Dunal }\end{array}$ & Prodr. [A. P. de Candolle] 13(1): 284. 1852. & Leptostemonum & Old World-Africa \\
\hline $\begin{array}{l}\text { Solanum heinianum D'Arcy \& } \\
\text { R.C.Keating }\end{array}$ & Phytologia 34: 282. 1976. & Leptostemonum & $\begin{array}{l}\text { Old World- } \\
\text { Madagascar }\end{array}$ \\
\hline Solanum herculeum Bohs & Plant Syst. Evol. 228: 44. 2001. & Normania & \\
\hline Solanum humblotii Dammer & Bot. Jahrb. Syst. 38: 184. 1906. & $\begin{array}{l}\text { African non-spiny } \\
\text { (ANS) }\end{array}$ & \\
\hline Solanum humile Lam. & Tabl. Encycl. 2: 23. 1794. & Leptostemonum & Old World-Africa \\
\hline Solanum imamense Dunal & Prodr. [A. P. de Candolle] 13(1): 85. 1852. & $\begin{array}{l}\text { African non-spiny } \\
\text { (ANS) }\end{array}$ & \\
\hline Solanum inaequiradians Werderm. & $\begin{array}{l}\text { Notizbl. Bot. Gart. Berlin-Dahlem 12: } 90 . \\
1934 .\end{array}$ & Leptostemonum & Old World-Africa \\
\hline Solanum incanum L. & Sp. Pl. 188. 1753. & Leptostemonum & Old World-Africa \\
\hline Solanum insanum $\mathrm{L}$. & Mant. 1: 46. 1767. & Leptostemonum & $\begin{array}{l}\text { Old World-Tropical } \\
\text { Asia }\end{array}$ \\
\hline Solanum ivohibe D'Arcy \& Rakot. & Fl. Madag., Fam. 176: 97. 1994. & $\begin{array}{l}\text { African non-spiny } \\
\text { (ANS) }\end{array}$ & \\
\hline Solanum jubae Bitter & Bot. Jahrb. Syst. 54: 501. 1917. & Leptostemonum & Old World-Africa \\
\hline Solanum laciniatum Aiton & Hort. Kew. ed. 1, 1: 247. 1789. & Archaesolanum & \\
\hline Solanum lamprocarpum Bitter & $\begin{array}{c}\text { Repert. Spec. Nov. Regni Veg. Beih. 16: } 107 . \\
1923 .\end{array}$ & Leptostemonum & Old World-Africa \\
\hline Solanum lanzae J.-P.Lebrun \& Stork & Candollea 50: 217.1995. & Leptostemonum & Old World-Africa \\
\hline Solanum lichtensteinii Willd. & Enum. Pl. (Willdenow) 1: 238. 1809. & Leptostemonum & Old World-Africa \\
\hline Solanum lidii Sunding & Blyttia 24: 368. 1966. & Leptostemonum & Old World \\
\hline $\begin{array}{l}\text { Solanum linnaeanum Hepper \& } \\
\text { P.-M.L. Jaeger }\end{array}$ & Kew Bull. 41: 435. 1986. & Leptostemonum & Old World-Africa \\
\hline Solanum litoraneum A.E.Gonç. & Kew Bull. 52(3): 703. 1997. & Leptostemonum & Old World-Africa \\
\hline Solanum lycopersicum $\mathrm{L}$. & Sp. Pl. 185. 1753. & Potato & Tomato \\
\hline Solanum macracanthum A.Rich. & Tent. Fl. Abyss. 2: 106. 1850. & Leptostemonum & Old World-Africa \\
\hline Solanum macrocarpon $\mathrm{L}$. & Mant. Pl. Altera: 205. 1771. & Leptostemonum & Old World-Africa \\
\hline Solanum macrothyrsum Dammer & Bot. Jahrb. Syst. 38: 185. 1906. & $\begin{array}{l}\text { African non-spiny } \\
\text { (ANS) }\end{array}$ & \\
\hline Solanum madagascariense Dunal & Prodr. [A. P. de Candolle] 13(1): 99. 1852. & $\begin{array}{l}\text { African non-spiny } \\
\text { (ANS) }\end{array}$ & \\
\hline $\begin{array}{l}\text { Solanum mahoriense } \\
\text { D'Arcy \& Rakot. }\end{array}$ & Ann. Missouri Bot. Gard. 73: 498. 1986. & Leptostemonum & $\begin{array}{l}\text { Old World- } \\
\text { Madagascar }\end{array}$ \\
\hline Solanum malindiense Voronts. & Syst. Bot. 35: 904. 2010. & Leptostemonum & Old World-Africa \\
\hline Solanum mammosum L. & Sp. Pl. 187. 1753. & Leptostemonum & Acanthophora \\
\hline Solanum marginatum L.f. & Suppl. 147. 1781. & Leptostemonum & Old World-Africa \\
\hline Solanum mauense Bitter & $\begin{array}{c}\text { Repert. Spec. Nov. Regni Veg. Beih. 16: } 42 . \\
1923 .\end{array}$ & Leptostemonum & Old World-Africa \\
\hline Solanum mauritianum Scop. & Delic. Fl. Faun. Insubr. 3: 16. 1788. & Brevantherum & \\
\hline Solanum melastomoides C.H.Wright & Bull. Misc. Inform. Kew 1894: 128. 1894. & Leptostemonum & Old World-Africa \\
\hline Solanum melongena $\mathrm{L}$. & Sp. Pl. 186. 1753. & Leptostemonum & $\begin{array}{l}\text { Old World-Tropical } \\
\text { Asia }\end{array}$ \\
\hline Solanum memphiticum J.F.Gmel. & Syst. Nat., ed. 13[bis] 2(1): 385. 1791 & Morelloid & Black nightshade \\
\hline Solanum muricatum Aiton & Hort. Kew, ed. 1, 1: 250. 1789. & Potato & Basarthrum \\
\hline Solanum myoxotrichum Baker & J. Linn. Soc., Bot. 21: 426. 1885. & Leptostemonum & $\begin{array}{l}\text { Old World- } \\
\text { Madagascar }\end{array}$ \\
\hline $\begin{array}{l}\text { Solanum myrsinoides D'Arcy \& } \\
\text { Rakot. }\end{array}$ & Fl. Madag., Fam. 176: 115. 1994. & $\begin{array}{l}\text { African non-spiny } \\
\text { (ANS) }\end{array}$ & \\
\hline Solanum nava Webb \& Berthel. & Phyt. Canar. 2. 3(3): 123. 1845. & Normania & \\
\hline
\end{tabular}




\begin{tabular}{|c|c|c|c|}
\hline Species & Place of original publication & Major Clade & Minor clade \\
\hline Solanum nigriviolaceum Bitter & $\begin{array}{c}\text { Repert. Spec. Nov. Regni Veg. Beih. 16: } 163 . \\
1923 .\end{array}$ & Leptostemonum & Old World-Africa \\
\hline Solanum nigrum $\mathrm{L}$. & Sp. Pl. 186. 1753. & Morelloid & Black nightshade \\
\hline Solanum nitidibaccatum Bitter & Repert. Spec. Nov. Regni Veg. 11: 208. 1912. & Morelloid & Black nightshade \\
\hline Solanum pampaninii Chiov. & Res. Sci. Somalia Ital. 1: 128. 1916. & Leptostemonum & Old World-Africa \\
\hline Solanum pauperum C.H.Wright & Bull. Misc. Inform. Kew 1894: 127. 1894. & Leptostemonum & Old World-Africa \\
\hline Solanum pectinatum Dunal & Prodr. [A. P. de Candolle] 13(1): 250. 1852. & Leptostemonum & Lasiocarpa \\
\hline Solanum phoxocarpum Voronts. & Syst. Bot. 35: 903. 2010 & Leptostemonum & Old World-Africa \\
\hline Solanum pimpinellifolium L. & Cent. Pl. 1: 8. 1755. & Potato & Tomato \\
\hline Solanum polhillii Voronts. & Syst. Bot. 35: 902. 2010. & Leptostemonum & Old World-Africa \\
\hline $\begin{array}{l}\text { Solanum pseudospinosum } \\
\text { C.H.Wright }\end{array}$ & Fl. Trop. Afr. [Oliver et al.] 4, 2: 220. 1906. & Morelloid & Black nightshade \\
\hline Solanum pyracanthos Lam. & Tabl. Encycl. 2: 21. 1794. & Leptostemonum & $\begin{array}{l}\text { Old World- } \\
\text { Madagascar }\end{array}$ \\
\hline Solanum retroflexum Dunal & Prodr. [A. P. de Candolle] 13(1): 50. 1852. & Morelloid & Black nightshade \\
\hline Solanum richardii Dunal & $\begin{array}{c}\text { Encycl. [J. Lamarck \& al.] Suppl. 3: } 775 . \\
1814 .\end{array}$ & Leptostemonum & Old World-Africa \\
\hline Solanum rigidum Lam. & Tabl. Encycl. 2: 23. 1794. & Leptostemonum & Old World-Africa \\
\hline Solanum robustum H.L.Wendl. & Flora 27: 784. 1844 & Leptostemonum & Erythrotrichum \\
\hline Solanum rubetorum Dunal & Prodr. [A. P. de Candolle] 13(1): 304.1852. & Leptostemonum & Old World-Africa \\
\hline Solanum runsoriense C.H.Wright & Uganda Prot. (H.H.Johnston) 1: 326. 1902. & $\begin{array}{l}\text { African non-spiny } \\
\text { (ANS) }\end{array}$ & \\
\hline Solanum ruvu Voronts. & J. E. Afr. Nat. Hist. 99: 230. (2010) 2011. & Leptostemonum & Old World-Africa \\
\hline $\begin{array}{l}\text { Solanum sambiranense } \\
\text { D'Arcy \& Rakot. }\end{array}$ & Fl. Madag., Fam. 176: 123. 1994. & $\begin{array}{l}\text { African non-spiny } \\
\text { (ANS) }\end{array}$ & \\
\hline Solanum sarrachoides Sendtn. & $\begin{array}{l}\text { Fl. Bras. (Martius) 10: 18, tab. 1, fig. 1-8. } \\
1846 .\end{array}$ & Morelloid & Black nightshade \\
\hline Solanum scabrum Mill. & Gard. Dict. ed. 8, no. 6. 1768. & Morelloid & Black nightshade \\
\hline $\begin{array}{l}\text { Solanum schimperianum Hochst. } \\
\text { ex A.Rich. }\end{array}$ & Tent. Fl. Abyss. 2: 98. 1850. & Leptostemonum & Old World-Africa \\
\hline Solanum schliebenii Werderm. & $\begin{array}{l}\text { Notizbl. Bot. Gart. Berlin-Dahlem 12: } 92 . \\
1934 .\end{array}$ & Leptostemonum & Old World-Africa \\
\hline Solanum schumannianum Dammer & $\begin{array}{c}\text { Pflanzenw. Ost-Afrikas C (Engler): } 352 . \\
1895 .\end{array}$ & Leptostemonum & Old World-Africa \\
\hline Solanum setaceum Dammer & Pflanzenw. Ost-Afrikas C (Engler): 33. 1895. & Leptostemonum & Old World-Africa \\
\hline Solanum sisymbriifolium Lam. & Tabl. Encycl. 2: 25. 1794. & Leptostemonum & Sisymbriifolium \\
\hline Solanum sodomeodes Kuntze & Revis. Gen. Pl. 3(3): 227. 1898. & Leptostemonum & Old World-Africa \\
\hline Solanum somalense Franch. & Sert. Somal. 47. 1882. & Leptostemonum & Old World-Africa \\
\hline Solanum stipitatostellatum Dammer & $\begin{array}{l}\text { Abh. Königl. Akad. Wiss. Berlin 1894: } 63 . \\
1894 .\end{array}$ & Leptostemonum & Old World-Africa \\
\hline Solanum supinum Dunal & Prodr. [A. P. de Candolle] 13(1): 289. 1852. & Leptostemonum & Old World-Africa \\
\hline Solanum taitense Vatke & Linnaea 43: 327.1882. & Leptostemonum & Old World-Africa \\
\hline Solanum tarderemotum Bitter & Repert. Spec. Nov. Regni Veg. 10: 547. 1912. & Morelloid & Black nightshade \\
\hline Solanum terminale Forssk. & Fl. Aegypt.-Arab. 45. 1775. & $\begin{array}{l}\text { African non-spiny } \\
\text { (ANS) }\end{array}$ & \\
\hline Solanum tettense Klotzsch & $\begin{array}{l}\text { Naturw. Reise Mossambique (Peters) 1: } 237 . \\
1861 .\end{array}$ & Leptostemonum & Old World-Africa \\
\hline Solanum thomsonii C.H.Wright & Fl. Trop. Afr. [Oliver et al.] 4, 2: 217. 1906. & Leptostemonum & Old World-Africa \\
\hline Solanum toliaraea D'Arcy \& Rakot. & Ann. Missouri Bot. Gard. 76: 351. 1989. & Leptostemonum & $\begin{array}{l}\text { Old World- } \\
\text { Madagascar }\end{array}$ \\
\hline Solanum tomentosum $\mathrm{L}$. & Sp. Pl. 188. 1753. & Leptostemonum & Old World-Africa \\
\hline Solanum torreanum A.E.Gonç. & Kew Bull., 52(3): 706. 1997. & Leptostemonum & Old World-Africa \\
\hline Solanum torvum $\mathrm{Sw}$. & Prodr. [O. P. Swartz] 47. 1788. & Leptostemonum & Torva \\
\hline $\begin{array}{l}\text { Solanum trichopetiolatum D'Arcy } \\
\text { \& Rakot. }\end{array}$ & Fl. Madag., Fam. 176: 130. 1994. & $\begin{array}{l}\text { African non-spiny } \\
\text { (ANS) }\end{array}$ & \\
\hline Solanum triflorum Nutt. & Gen. N. Amer. Pl. 1: 128. 1818. & Morelloid & \\
\hline
\end{tabular}




\begin{tabular}{|c|c|c|c|}
\hline Species & Place of original publication & Major Clade & Minor clade \\
\hline Solanum trisectum Dunal & Prodr. [A. P. de Candolle] 13(1): 36. 1852. & Normania & \\
\hline Solanum truncicola Bitter & Bot. Jahrb. Syst. 54: 435.1917. & $\begin{array}{l}\text { African non-spiny } \\
\text { (ANS) }\end{array}$ & \\
\hline Solanum tuberosum $\mathrm{L}$. & Sp. Pl. 185. 1753. & Potato & Petota \\
\hline Solanum umalilaense Manoko & PhytoKeys 16: 67. 2012. & Morelloid & Black nightshade \\
\hline $\begin{array}{l}\text { Solanum umtuma Voronts. \& } \\
\text { S.Knapp }\end{array}$ & PhytoKeys 8: 4. 2012. & Leptostemonum & Old World-Africa \\
\hline $\begin{array}{l}\text { Solanum usambarense Bitter \& } \\
\text { Dammer }\end{array}$ & $\begin{array}{c}\text { Repert. Spec. Nov. Regni Veg. Beih. 16: } 40 . \\
1923 .\end{array}$ & Leptostemonum & Old World-Africa \\
\hline Solanum usaramense Dammer & $\begin{array}{c}\text { Pflanzenw. Ost-Afrikas C (Engler): } 353 . \\
1895 .\end{array}$ & Leptostemonum & Old World-Africa \\
\hline Solanum vespertilio Aiton & Hort. Kew. ed. 1, 1: 252. 1789. & Leptostemonum & Old World \\
\hline Solanum viarum Dunal & Prodr. [A. P. de Candolle] 13(1): 240. 1852. & Leptostemonum & Acanthophora \\
\hline Solanum villosum Mill. & Gard. Dict. ed. 8, no. 2. 1768. & Morelloid & Black nightshade \\
\hline Solanum violaceum Ortega & Nov. Pl. Descr. Dec. 56. 1798. & Leptostemonum & $\begin{array}{l}\text { Old World-Tropical } \\
\text { Asia }\end{array}$ \\
\hline Solanum virginianum $\mathrm{L}$. & Sp. Pl. 187. 1753. & Leptostemonum & $\begin{array}{c}\text { Old World-Tropical } \\
\text { Asia }\end{array}$ \\
\hline Solanum wendlandii Hook.f. & Bot. Mag. 113: tab. 6914. 1887. & $\begin{array}{l}\text { Wendlandii- } \\
\text { Allophyllum }\end{array}$ & \\
\hline Solanum wittei Robyns & Bull. Jard. Bot. État Bruxelles 17: 82. 1943. & Leptostemonum & Old World-Africa \\
\hline Solanum wrightii Benth. & Fl. Hongk. 243. 1861. & Leptostemonum & Androceras-Crinitum \\
\hline Solanum zanzibarense Vatke & Linnaea 43: 326. 1882. & Leptostemonum & Old World-Africa \\
\hline
\end{tabular}

Table 2. Country distribution of Solanum species in Africa (as defined here); introduced (incl. cultivated) species in brackets (epithet); taxa not included in the keys because they are known from a singleton cultivated specimen, are in italic type. All records based on specimens examined by the authors with verified identities. The status of $S$. torvum is not completely clear, but it is most likely to be introduced from the New World, so is treated as that here; $S$. americanum, on the other hand, appears to have a worldwide distribution, so is treated as native. The occurrence of $S$. rigidum in Senegal is doubtful, the specimen is very old and the label may be in error. Cultivated plants are often not collected, so the absence of records of commonly cultivated crops (e.g. S. lycopersicum, S. macrocarpon, S. tuberosum) should not be interpreted as lack of occurrence, merely as lack of collections. Solanum diphyllum was recorded from Eygpt by Fawzi and Habeeb (2016) with a verifiable photograph; this Mexican species is widely cultivated and easily naturalised and is likely to be spreading around the Mediterranean.

\begin{tabular}{|c|c|}
\hline Country & Species \\
\hline Algeria & herculeum, linnaeanum, nigrum, villosum \\
\hline Angola & $\begin{array}{l}\text { aculeastrum. (aculeatissimum), aethiopicum, americanum, anguivi, anomalum, aureitomentosum, (betaceum), } \\
\text { campylacanthum, capsicoides, catombelense, dasyphyllum, humile, lichtensteinii, (bcopersicum), macrocarpon, } \\
\text { mammosum, (mauritianum), pauperum, scabrum, tarderemotum, terminale, tettense, villosum }\end{array}$ \\
\hline Azores & (linnaeanum), (chenopodioides), (chrysotrichum), (nava?), nigrum, (maritianum), (pseudocapsicum), villosum \\
\hline Benin & anguivi, anomalum, incanum, scabrum, (torvum) \\
\hline Botswana & campylacanthum, catombelense, lichtensteinii, retroflexum, scabrum, supinum, tarderemotum, tettense, villosum \\
\hline Burkina Faso & cerasiferum, dasyphyllum, incanum, scabrum \\
\hline Burundi & $\begin{array}{l}\text { aculeastrum, anguivi, campylacanthum, cyaneopurpureum, dasyphyllum, mammosum, memphiticum, } \\
\text { tarderemotum, terminale, villosum }\end{array}$ \\
\hline Cabo Verde & (agrarium), americanum, (lycopersicum), nigrum, rigidum, scabrum, tarderemotum, (torvum) \\
\hline Cameroon & $\begin{array}{l}\text { aculeastrum, (aculeatissimum), aethiopicum, americanum, anguivi, anomalum, cerasiferum, dasyphyllum, } \\
\text { (erianthum), giganteum, (lycopersicum), macrocarpon, (mauritianum), (melongena), pseudospinosum, scabrum, } \\
\text { tarderemotum, terminale, (torvum), (wendlandii), (wrightii) }\end{array}$ \\
\hline
\end{tabular}




\begin{tabular}{|c|c|}
\hline Country & Species \\
\hline $\begin{array}{l}\text { Canary Islands } \\
\text { (Spain) }\end{array}$ & $\begin{array}{l}\text { americanum, (laxum), (lycopersicum), (mauritianum), nava, nigrum, (pseudocapsicum), (robustum), } \\
\text { vespertilio, (wendlandii) }\end{array}$ \\
\hline $\begin{array}{l}\text { Central African } \\
\text { Republic (CAR) }\end{array}$ & $\begin{array}{l}\text { aculeastrum, (aculeatissimum), anguivi, cerasiferum, dasyphyllum, giganteum, (lycopersicum), macrocarpon, } \\
\text { scabrum, (seaforthianum), terminale, (torvum), (wrightii) }\end{array}$ \\
\hline Chad & cerasiferum, forskalii, incanum, tarderemotum, villosum \\
\hline $\begin{array}{l}\text { Comoros (incl. } \\
\text { Mayotte) }\end{array}$ & americanum, macrothyrsum, richardii, scabrum, tarderemotum, terminale, (torvum) \\
\hline $\begin{array}{l}\text { Democratic } \\
\text { Republic of the } \\
\text { Congo }\end{array}$ & $\begin{array}{l}\text { aculeastrum, (aculeatissimum), aethiopicum, anomalum, aureitomentosum, campylacanthum, cerasiferum, } \\
\text { (chrysotrichum), cyaneopurpureum, dasyphyllum, giganteum, lichtensteinii, (lycopersicum), macrocarpon, } \\
\text { (mammosum), (mauritianum), (melongena), memphiticum, richardii, runsoriense, scabrum, (seaforthianum), } \\
\text { tarderemotum, terminale, tettense, (torvum), (viarum), wittei, (wrightii) }\end{array}$ \\
\hline $\begin{array}{l}\text { Republic of the } \\
\text { Congo }\end{array}$ & aculeastrum, anomalum, dasyphyllum, (lycopersicum), terminale, (torvum) \\
\hline Cote d'Ivoire & $\begin{array}{l}\text { (aculeatissimum), americanum, anguivi, anomalum, cerasiferum, dasyphyllum, (lycopersicum), scabrum, } \\
\text { terminale, (torvum) }\end{array}$ \\
\hline Djibouti & somalense \\
\hline $\begin{array}{l}\text { Egypt (incl. Hala'ib } \\
\text { triangle)* }\end{array}$ & $\begin{array}{l}\text { coagulans, (diphyllum), dulcamara, elaeagnifolium, forskalii, incanum, (lycopersicum), macrocarpon, } \\
\text { (melongena), memphiticum, nigrum, scabrum, (torvum), villosum, virginianum, (wendlandii), (wrightii) }\end{array}$ \\
\hline Equatorial Guinea & $\begin{array}{l}\text { (aculeatissimum), aethiopicum, americanum, anguivi, dasyphyllum, giganteum, (lycopersicum), } \\
\text { pseudospinosum, scabrum, terminale, (torvum) }\end{array}$ \\
\hline Eritrea & $\begin{array}{l}\text { adoense, americanum, anguivi, campylacanthum, cerasiferum, coagulans, dasyphyllum, forskalii, glabratum, } \\
\text { incanum, (lycopersicum), macracanthum, marginatum, melastomoides, (melongena), memphiticum, } \\
\text { muricatum, scabrum, schimperianum, somalense, tarderemotum, terminale, villosum }\end{array}$ \\
\hline Ethiopia & $\begin{array}{l}\text { (aculeatissimum), adoense, americanum, anguivi, arundo, campylacanthum, capsicoides, cerasiferum, } \\
\text { coagulans, cordatum, dennekense, forskalii, giganteum, glabratum, hastifolium, hirtulum, incanum, jubae, } \\
\text { lanzae, (bycopersicum), macracanthum, macrocarpon, marginatum, melastomoides, memphiticum, muricatum, } \\
\text { pampaninii, runsoriense, schimperianum, somalense, tarderemotum, terminale, tettense, villosum, (wrightii) }\end{array}$ \\
\hline Gabon & $\begin{array}{l}\text { aethiopicum, americanum, anguivi, anomalum, dasyphyllum, giganteum, macrocarpon, scabrum, terminale, } \\
\text { (torvum), (wrightii) }\end{array}$ \\
\hline Gambia & americanum, anguivi, cerasiferum, dasyphyllum \\
\hline Ghana & $\begin{array}{l}\text { (aculeatissimum), americanum, anguivi, anomalum, capsicoides, dasyphyllum, (erianthum), incanum, } \\
\text { macrocarpon, (melongena), scabrum, tarderemotum, terminale, (torvum), (wrightii) }\end{array}$ \\
\hline Guinea & (aculeatissimum), anguivi, (erianthum), scabrum, tarderemotum, terminale, (torvum), (wrightii) \\
\hline Guinea-Bissau & americanum, anguivi, cerasiferum, dasyphyllum, terminale \\
\hline Kenya & $\begin{array}{l}\text { aculeastrum, (aculeatissimum), aethiopicum, agnewiorum, americanum, anguivi, arundo, (betaceum), } \\
\text { campylacanthum, coagulans, cordatum, dasyphyllum, dennekense, forskalii, giganteum, goetzei, hastifolium, } \\
\text { incanum, jubae, lanzae, (laxum), (lycopersicum), macrocarpon, malindiense, mammosum, mauense, } \\
\text { (mauritianum), melastomoides, (melongena), nigriviolaceum, pampaninii, phoxocarpum, polhillii, } \\
\text { (pseudocapsicum), richardii, runsoriense, schumannianum, (seaforthianum), setaceum, sisymbrifolium, } \\
\text { somalense, stipitatostellatum, taitense, tarderemotum, terminale, tettense, (tuberosum), usambarense, usaramense, } \\
\text { villosum, (wendlandii), (wrightii), zanzibarense }\end{array}$ \\
\hline Lesotho & (aculeatissimum), (chenopodioides), lichtensteinii, retroflexum, scabrum, sodomeodes, tarderemotum \\
\hline Liberia & $\begin{array}{l}\text { (aculeatissimum), americanum, anguivi, anomalum, dasyphyllum, (lycopersicum), (mauritianum), scabrum, } \\
\text { terminale, (torvum) }\end{array}$ \\
\hline Libya & linnaeanum, nigrum, villosum, virginianum \\
\hline Madagascar & $\begin{array}{l}\text { aethiopicum, americanum, anguivi, batoides, (betaceum), betroka, bumeliifolium, croatii, erythracanthum, } \\
\text { heinianum, humblotii, imamense, insanum, ivohibe, (lycopersicum), macrocarpon, madagascariense, } \\
\text { mahoriense, (mauritianum), (melongena), myoxotrichum, myrsinoides, (pseudocapsicum), pyracanthos, } \\
\text { richardii, sambiranense, scabrum, (seaforthianum), tarderemotum, toliaraea, (torvum), trichopetiolatum, } \\
\text { truncicola, (tuberosum), violaceum }\end{array}$ \\
\hline Madeira (Portugal) & $\begin{array}{l}\text { (chenopodioides), dulcamara, (laxum), linnaeanum, (lycopersicum), marginatum, nigrum, (pseudocapsicum), } \\
\text { trisectum, villosum }\end{array}$ \\
\hline Malawi & $\begin{array}{l}\text { aculeastrum, (aculeatissimum), aethiopicum, americanum, anguivi, aureitomentosum, campylacanthum, } \\
\text { (chrysotrichum), dasyphyllum, giganteum, goetzei, lichtensteinii, macrocarpon, retroflexum, richardii, scabrum, } \\
\text { schumannianum, (seaforthianum), tarderemotum, terminale, tettense, (torvum), villosum, (wendlandii), } \\
\text { (wrightii) }\end{array}$ \\
\hline Mali & cerasiferum, dasyphyllum, forskalii, incanum, (bycopersicum), tarderemotum \\
\hline
\end{tabular}




\begin{tabular}{|c|c|}
\hline Country & Species \\
\hline Mauritania & dasyphyllum, scabrum, villosum \\
\hline $\begin{array}{l}\text { Mauritius (incl. La } \\
\text { Réunion) }\end{array}$ & $\begin{array}{l}\text { americanum, (anguivi), (chenopodioides), erythracanthum, insanum, (lycopersicum), (mauritianum), } \\
\text { (melongena), richardii, tarderemotum, (torvum), violaceum }\end{array}$ \\
\hline Morocco & dulcamara, elaeagnifolium, forskalii, herculeum, (laciniatum), linnaeanum, nigrum, triflorum, villosum \\
\hline Mozambique & $\begin{array}{l}\text { aculeastrum, (aculeatissimum), aethiopicum, americanum, anguivi, aureitomentosum, campylacanthum, } \\
\text { catombelense, dasyphyllum, giganteum, goetzei, lamprocarpum, lichtensteinii, linnaeanum, litoraneum, } \\
\text { retroflexum, richardii, scabrum, stipitatostellatum, tarderemotum, tettense, torreanum, (torvum), usaramense, } \\
\text { (viarum), villosum, zanzibarense }\end{array}$ \\
\hline Namibia & $\begin{array}{l}\text { burchellii, campylacanthum, capense, catombelense, elaeagnifolium, numile, lichtensteinii, (lycopersicum), } \\
\text { pimpinellifolium, retroflexum, scabrum, (seaforthianum), supinum, tarderemotum, tettense }\end{array}$ \\
\hline Niger & anguivi, forskalii, incanum, (lycopersicum), villosum \\
\hline Nigeria & $\begin{array}{l}\text { aculeastrum, (aculeatissimum), aethiopicum, americanum, anguivi, anomalum, cerasiferum, dasyphyllum, } \\
\text { (erianthum), giganteum, incanum, (lycopersicum), macrocarpon, melongena, scabrum, terminale, (torvum), } \\
\text { villosum, (wrightii) }\end{array}$ \\
\hline Rwanda & $\begin{array}{l}\text { aculeastrum, (aculeatissimum), anguivi, campylacanthum, cyaneopurpureum, dasyphyllum, giganteum, } \\
\text { tarderemotum, terminale, wittei }\end{array}$ \\
\hline Sáo Tome e Principe & americanum, capsicoides, (melongena), scabrum, terminale \\
\hline Senegal & anguivi, cerasiferum, forskalii, incanum, (lycopersicum), rigidum ?, scabrum, tarderemotum \\
\hline Seychelles & aldabrense, americanum, scabrum \\
\hline Sierra Leone & $\begin{array}{l}\text { aculeatissimum, americanum, anguivi, capsicoides, dasyphyllum, (erianthum), (lycopersicum), macrocarpon, } \\
\text { (melongena), scabrum, tarderemotum, terminale, (torvum), (wrightii) }\end{array}$ \\
\hline Somalia & $\begin{array}{l}\text { arundo, campylacanthum, coagulans, cordatum, cymbalariifolium, dasyphyllum, dennekense, forskalii, } \\
\text { glabratum, hastifolium, incanum, jubae, melastomoides, (melongena), memphiticum, pampaninii, } \\
\text { schimperianum, somalense, tarderemotum, tettense, villosum }\end{array}$ \\
\hline South Africa & $\begin{array}{l}\text { aculeastrum, (aculeatissimum), africanum, americanum, anguivi, burchellii, campylacanthum, capense, } \\
\text { catombelense, (chenopodioides), (chrysotrichum), dasyphyllum, elaeagnifolium, giganteum, guineense, humile, } \\
\text { (laxum), lichtensteinii, linnaeanum, (mauritianum), (pseudocapsicum), retroflexum, rubetorum, (sarrachoides), } \\
\text { (seaforthianum), sisymbrifolium, sodomeodes, supinum, tarderemotum, terminale, tettense, tomentosum, } \\
\text { torreanum, (torvum), triflorum, (viarum), (wrightii) }\end{array}$ \\
\hline South Sudan & $\begin{array}{l}\text { aculeastrum, (aculeatissimum), aethiopicum, anguivi, campylacanthum, cerasiferum, coagulans, dasyphyllum, } \\
\text { giganteum, hastifolium, scabrum, tarderemotum, terminale }\end{array}$ \\
\hline $\begin{array}{l}\text { Sudan (incl. Hala'ib } \\
\text { triangle)* }\end{array}$ & $\begin{array}{l}\text { aculeastrum, adoense, aethiopicum, campylacanthum, cerasiferum, coagulans, forskalii, hastifolium, incanum, } \\
\text { macrocarpon, memphiticum, nigrum, scabrum, schimperianum, somalense, tarderemotum, villosum }\end{array}$ \\
\hline Swaziland & $\begin{array}{l}\text { aculeastrum, campylacanthum, catombelense, retroflexum, (robustum), (seaforthianum), sisymbriifolium, } \\
\text { torreanum }\end{array}$ \\
\hline Tanzania & $\begin{array}{l}\text { aculeastrum, (aculeatissimum), americanum, anguivi, arundo, (atropurpureum), aureitomentosum, } \\
\text { (betaceum), campylacanthum, coagulans, cyaneopurpureum, dasyphyllum, dennekense, giganteum, goetzei, } \\
\text { hastifolium, inaequiradians, lamprocarpum, lanzae, lichtensteinii, (lycopersicum), macrocarpon, mauense, } \\
\text { (melongena), memphiticum, (pectinatum), phoxocarpum, polhillii, richardii, (robustum), scabrum, schliebenii, } \\
\text { schumannianum, (seaforthianum), setaceum, stipitatostellatum, taitense, tarderemotum, terminale, tettense, } \\
\text { thomsonii, (tuberosum), umalilaense, usambarense, usaramense, villosum, (wendlandii), wittei, (wrightii), } \\
\text { zanzibarense }\end{array}$ \\
\hline Togo & (aculeatissimum), aethiopicum, americanum, anguivi, anomalum, (melongena), scabrum, terminale, (torvum) \\
\hline Tunisia & linnaeanum, (lycopersicum), nigrum, triflorum, villosum \\
\hline Uganda & $\begin{array}{l}\text { aculeastrum, (aculeatissimum), aethiopicum, americanum, anguivi, (betaceum), campylacanthum, } \\
\text { cerasiferum, coagulans, cyaneopurpureum, dasyphyllum, giganteum, hastifolium, lanzae, macrocarpon, } \\
\text { mammosum, mauense, mauritianum, memphiticum, runsoriense, scabrum, schumannianum, (seaforthianum), } \\
\text { tarderemotum, terminale, tettense, villosum, wittei, (wrightii) }\end{array}$ \\
\hline Western Sahara & villosum \\
\hline Zambia & $\begin{array}{l}\text { (aculeatissimum), americanum, anguivi, aureitomentosum, campylacanthum, (chrysotrichum), goetzei, } \\
\text { lichtensteinii, (lycopersicum), retroflexum, richardii, scabrum, (seaforthianum), tarderemotum, terminale, } \\
\text { tettense, }(\text { torvum }),(\text { tuberosum }), \text { villosum, (wendlandii), (wrightii) }\end{array}$ \\
\hline Zimbabwe & $\begin{array}{l}\text { aculeastrum. (aculeatissimum), anguivi, aureitomentosum, (betaceum), campylacanthum, catombelense, } \\
\text { giganteum, lichtensteinii, linnaeanum, (mauritianum), retroflexum, richardii, scabrum, (seaforthianum), } \\
\text { tarderemotum, terminale, villosum, (wendlandii) }\end{array}$ \\
\hline
\end{tabular}

*Possession of the area known as the Hala'ib triangle is disputed between Egypt and Sudan, species occurring there are listed under both coutries. 


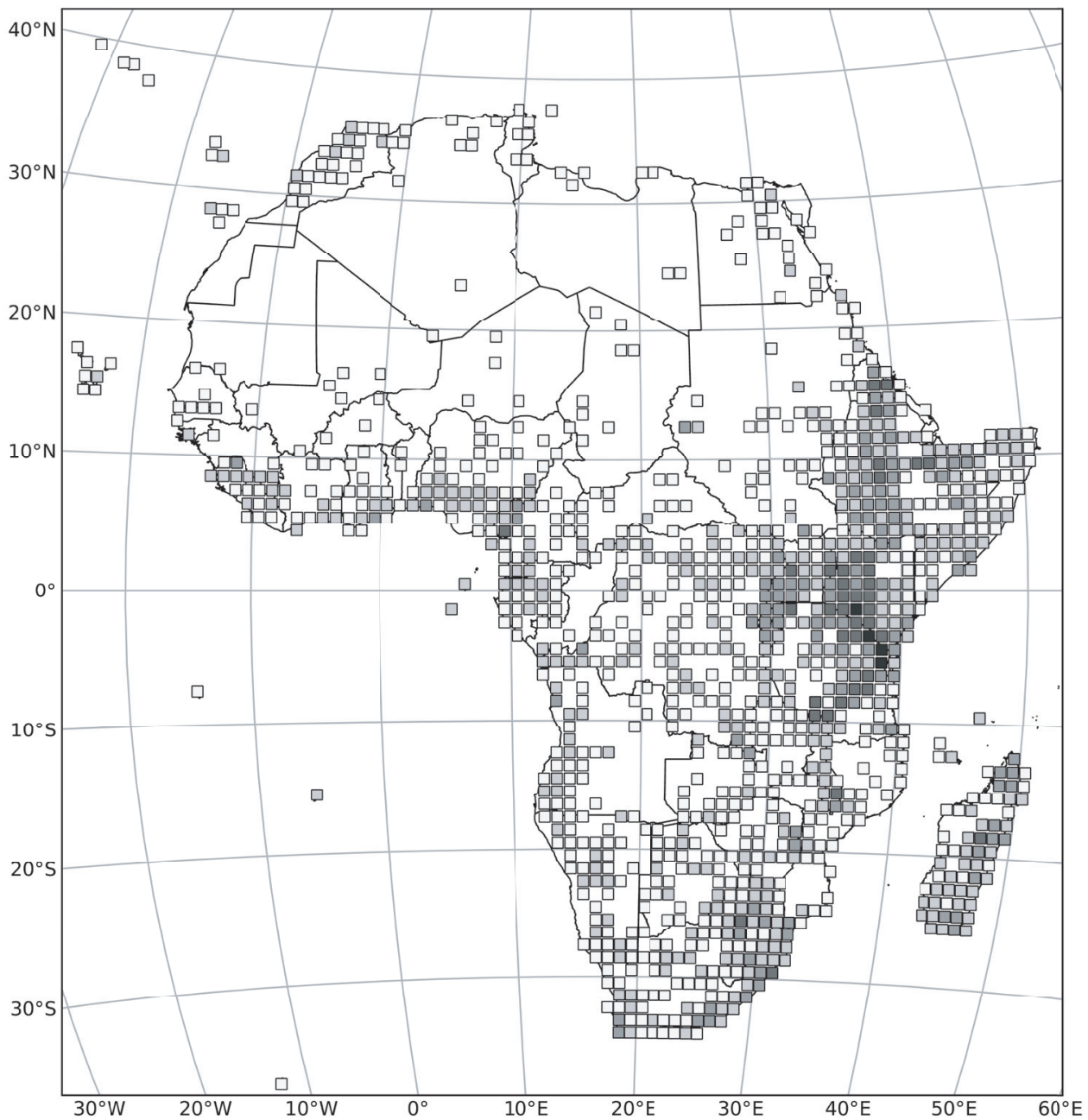

\section{Species richness}
$\square \quad 1-2$
ㄴ $2-6$
ㅁ $6-11$
- $11-20$
- $20-30$

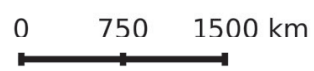

Figure I. Heat map of Solanum diversity in Africa. Darker degree squares indicate greater species richness. The middle to high elevation regions of eastern Africa (Kenya/Tanzania) have the highest high species diversity, followed by secondary areas of species richness in the Ethiopian plateaus, dry areas of central Madagascar, South Africa and the area around Mount Cameroon. We have not analysed how collecting effort has influenced these patterns, but it is likely to be important. As the Leptostemonum Clade has the largest number of species in Africa, diversity in that clade drives species richness overall (see Vorontsova and Knapp 2016, figure 2). Map prepared by Sarah Ficinski. 

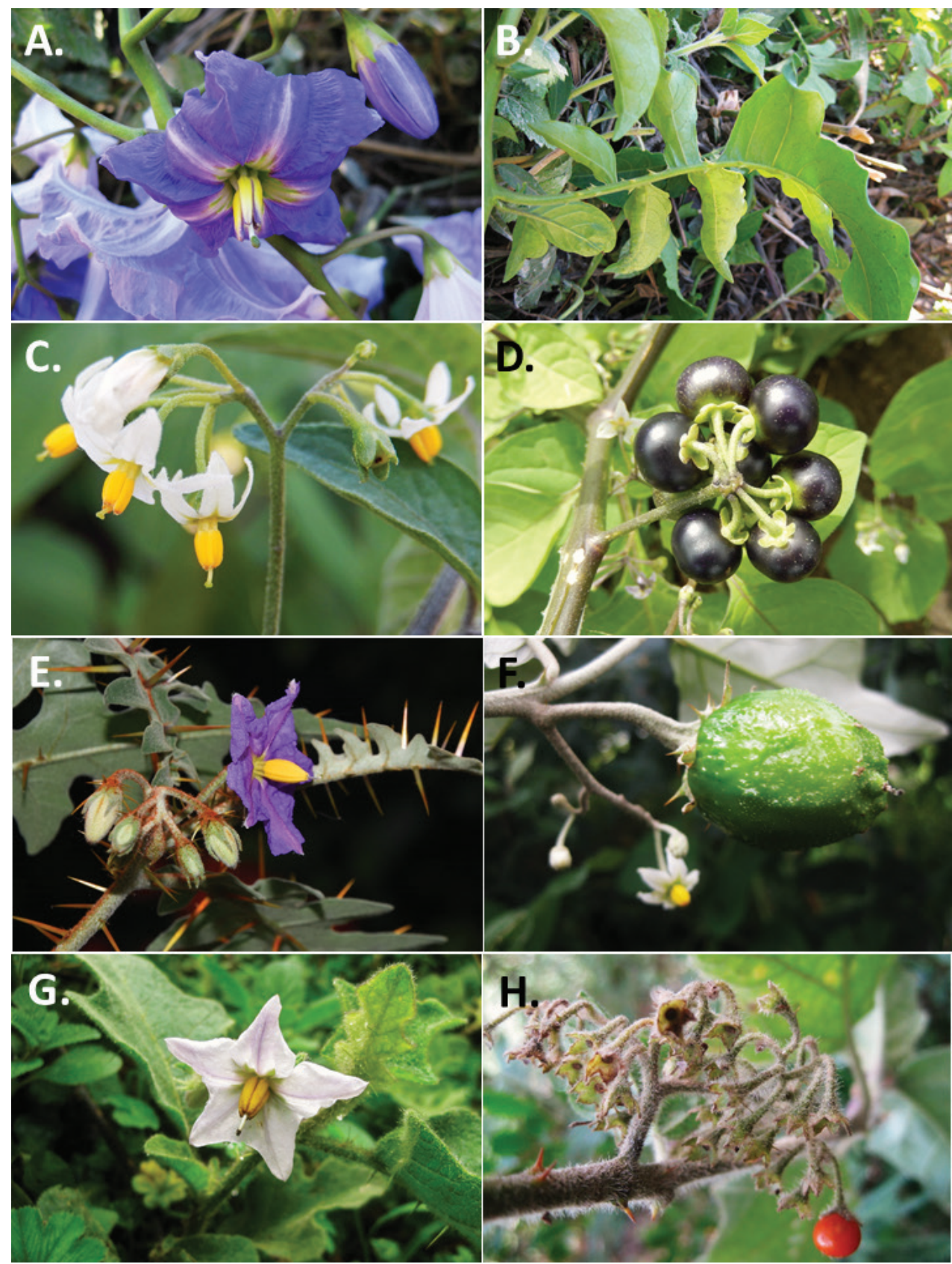

Figure 2. A, B Solanum wendlandii Hook.f. (Allophyllum-Wendlandii Clade) C Solanum tarderemotum Bitter (Morelloid Clade) D Solanum scabrum Mill. (Morelloid Clade) E Solanum pyracanthos Lam. (Leptostemonum Clade) F Solanum aculeastrum Dunal (Leptostemonum Clade) G Solanum nigriviolaceum Bitter (Leptostemonum Clade) H Solanum usambarense Bitter \& Dammer (Leptostemonum Clade). Photos A, B, F, G H by M.S. Vorontosova C, D, E by S. Knapp. 


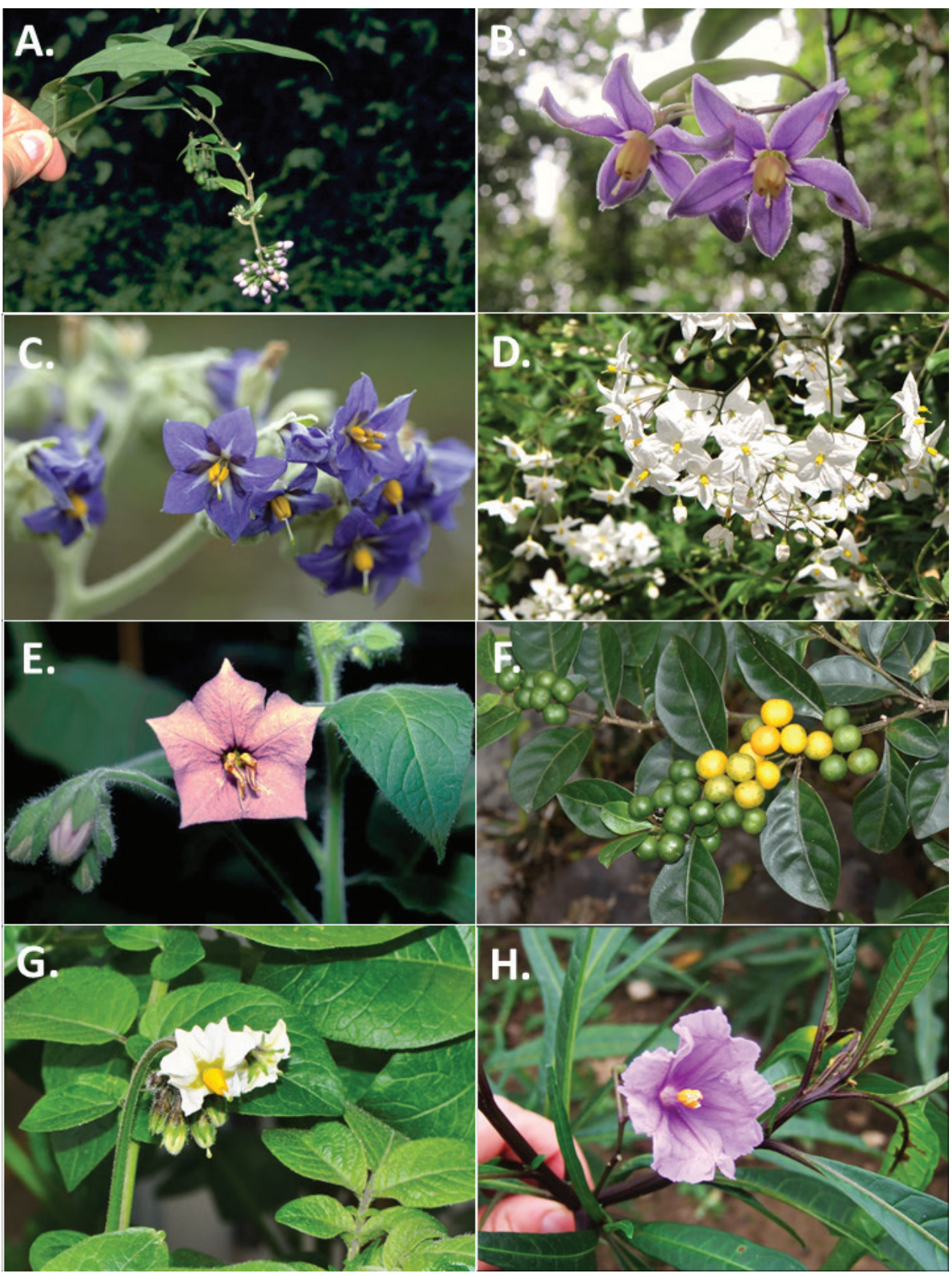

Figure 3. A Solanum terminale Forssk. (African non-spiny Clade) B Solanum madagascariense Dunal (African non-spiny Clade) C Solanum mauritianum Scop. (Brevantherum Clade) D Solanum laxum Spreng. (Dulcamaroid Clade) E Solanum trisectum Dunal (Normania Clade) F Solanum diphyllum L. (Geminata Clade) G Solanum tuberosum L. (Potato Clade) H Solanum laciniatum Aiton (Archaeosolanum Clade). Photos A, C, D, E, F, G, H by S. Knapp B by M.S. Vorontsova. 
Four species have been recorded from this area, for which we have only seen single specimens, all of which are cultivated and not naturalised. Solanum laciniatum Aiton (Archaesolanum Clade), the kangaroo apple from Australia and New Zealand, has been recorded from Morocco, S. agrarium Sendtn. (Leptostemonum Clade, section Acanthophora Dunal sensu Nee 1979) from Brazil has only recently been collected in the Cape Verde Islands and S. atropurpureum Schrank (Leptostemonum Clade, section Acanthophora Dunal sensu Nee 1979) from Brazil and S. pectinatum Dunal (Leptostemonum Clade, section Lasiocarpa Dunal sensu Whalen et al. 1981) from Mexico have been recorded from Tanzania in a botanical garden. These singletons have been included in Table 1, but not in the keys below; descriptions should be checked if identification is ambiguous.

Each species name is hyperlinked to its page on Solanaceae Source (www.solanaceaesource.org) where photographs (if available), descriptions and other information can be found. An expanded key to all of the thirteen major clades of Solanum worldwide is in preparation (R. Hilgenhof and T. Särkinen, pers. comm.). Instructions on how to use Solanaceae source are included in the Materials and Methods.

Key to the groups of Solanum in continental Africa, Madagascar (incl. the islands of Réunion, the Comoros and the Seychelles), Macaronesia and the Cape Verde Islands

1a Trichomes of stems and leaves stellate or echinoid.......................................2

1b Trichomes of stems and leaves simple (unbranched) or dendritically branched, never stellate or echinoid.......................................................................... 3

2a Anthers ellipsoid in outline; inflorescences many times branched; branching dichasial; stems without prickles

Key 6. Brevantherum Clade

2b Anthers tapering in outline; inflorescences branched or unbranched; branching monochasial; stems with or without prickles

Key 7. Leptostemonum Clade

3a Shrubs, small trees or woody vines ...................................................... 4

3b Herbs or if plants woody, only at the base; never true vines, occasionally scandent.

4a Stems with hooked prickles; anthers tapering

Solanum wendlandii Hook.f. (Wendlandii-Allophyllum Clade)

$4 \mathrm{~b} \quad$ Stems without prickles; anthers ellipsoid.

5a Small trees with foetid cordate leaves; flowers waxy pink or greenish white; anther connectives enlarged; fruit a large turbinate berry with fleshy pulp; cultivated tree tomato

Solanum betaceum Cav. (Pachyphylla [Cyphomandra] Clade)

$5 \mathrm{~b} \quad$ Shrubs or woody vines; leaves, flowers and anther connectives not as above. 
6a Small shrubs with paired (geminate) leaves; flowers nodding, white; fruit held on erect pedicels, orange; cultivated or occasionally naturalised in northern Africa.

$6 \mathrm{~b}$ Woody vines or scandent shrubs; leaves, flowers and fruit not as above; continental Africa, Madagascar; or cultivated

7a Leaves lanceolate, with at least some branched trichomes on new growth; leaves of a pair more or less the same shape; inflorescence usually with 2 flowers; berry dark orange; cultivated "Jerusalem cherry".

Solanum pseudocapsicum L. (Geminata Clade)

$7 \mathrm{~b} \quad$ Leaves ovate or elliptic, completely glabrous; leaves of a pair markedly different in shape; inflorescence with more than 2 flowers; berry pale orange; only recorded from Egypt

Solanum diphyllum L. (Geminata Clade)

8a Base of pedicel enclosed in a small sleeve of rhachis tissue usually more than $0.5 \mathrm{~mm}$ long; Mediterranean northern Africa; Macaronesia; if in other parts of Africa, cultivated.

Key 3. Dulcamaroid Clade

$8 \mathrm{~b} \quad$ Base of pedicel peg-like, sometimes enclosed in a small sleeve of rhachis tissue, if so the sleeve less than $0.5 \mathrm{~mm}$ long; Continental tropical Africa; Madagascar; native plants Key 1. African non-spiny (ANS) Clade

9a Leaves pinnate or deeply pinnatifid .............................................11

9b Leaves simple (at most the margins toothed) or at most ternate ................12

10a Fleshy prostrate herbs; leaves pinnatifid, the leaflets not distinct; inflorescences unbranched. Solanum triflorum Nutt. (Morelloid Clade)

10b Spreading herbs or herbaceous scramblers, not fleshy; leaves pinnate with distinct leaflets; inflorescences branched or less often unbranched

Key 5. Potato Clade

11a Anthers dimorphic, of different sizes and two of the five with horn-like projections; Macaronesia and northern Africa

Key 2. Normania Clade

11b Anthers equal in size and shape, if unequal only slightly so; widespread or cultivated 12

12a Trichomes simple with a single long terminal cell (bayonet hairs); fruit a large greenish berry with purple stripes (more than $3 \mathrm{~cm}$ diameter), with abundant solid mesocarp; herbaceous vine.

Solanum muricatum Aiton (Potato Clade)

12b Trichomes simple or branched; fruit variously coloured (usually less than 1 $\mathrm{cm}$ in diameter), with juicy mesocarp; annual or short-lived perennial herbs

Key 4. Morelloid Clade

Key I.African non-spiny (ANS) Clade (descriptions Knapp and Vorontsova 20I6)

1a Leaves glabrous on both surfaces.....................................................2

1b Leaves with at least some pubescence on either surface (this sometimes sparse along veins and midrib) 
2a Inflorescence few-flowered, unbranched (at most furcate in Solanum betroka)...3

2b Inflorescence many flowered, usually many times branched .....................5

3a Flowers appearing fasciculate and axillary; corolla usually somewhat campanulate; fruit orange; South Africa.

Solanum guineense L.

$3 \mathrm{~b} \quad$ Flowers not appearing fasciculate; corolla stellate, the petals spreading or reflexed; fruit colour green, black or not known, never orange; Madagascar.... 4

4a Leaves clustered on short shoots; calyx lobes deltate, not divided to base; dry forests ............................................. Solanum betroka D'Arcy \& Rakot.

$4 \mathrm{~b} \quad$ Leaves not clustered on short shoots; calyx lobes long triangular, divided to the base; wet forests ....................... Solanum truncicola D'Arcy \& Rakot.

5a Flowers or fruits (or pedicel scars) in tightly packed groups on individual branches (these sometimes very short and the inflorescence appearing spicate) Solanum terminale Forssk.

$5 b$

$6 \mathrm{a}$

$6 \mathrm{~b}$

$7 \mathrm{a}$

$7 b$

$8 \mathrm{a}$

$8 b$

$9 \mathrm{a}$

$9 \mathrm{~b}$

$10 \mathrm{a}$

$10 \mathrm{~b}$

$11 \mathrm{a}$

$11 \mathrm{~b}$

$12 \mathrm{a}$

$12 b$

$13 \mathrm{a}$

Leaves spaced along shoots; fruit purple or black; Madagascar

Solanum truncicola D’Arcy \& Rakot.

Flowers or fruits (or pedicel scars) in tightly packed groups on individual branches (these sometimes very short and the inflorescence appearing spicate) Solanum terminale Forssk.

Flowers spaced on the open inflorescence, often unevenly so

Leaves spaced along the stem

Anthers opening by pores that elongate with age; mountains of continental Africa

Solanum runsoriense C.H.Wright

Anthers opening by delineated pores that do not elongate with age; Madagas-

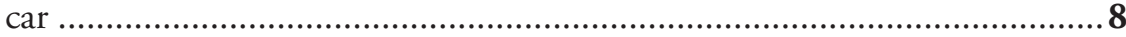

Leaves fleshy, thick and coriaceous, the venation not visible in dry specimens; fruit with thick pericarp (woody?)

Solanum myrsinoides D’Arcy \& Rakot.

Leaves membranous to coriaceous, not markedly thick and fleshy, the venation visible in dry specimens; fruit with thin pericarp, the seeds visible through the berry wall seeds 4-8 per berry; inflorescence axis thin and delicate...

Solanum trichopetiolatum D’Arcy \& Rakot. Petioles glabrous or with minute dendritic trichomes; seeds 20-40 per berry; inflorescence axis robust. Solanum madagascariense Dunal Leaf trichomes simple (unbranched)

Leaf trichomes branched (dendritic to echinoid) 16

Inflorescence axis unbranched, the flowers closely spaced 12

Inflorescence axis branched, often many times so 
$14 \mathrm{a}$ Stems strongly quadrangular; at least some leaves with shallow lobes; plants of seashore and dune habitats. Solanum africanum Mill.

$14 \mathrm{~b}$ Stems terete; leaves not lobed; plants of forests and forest edges..... 15

15a Leaf pubescence very sparse, confined to the midrib or near the petiole; flowers not heterostylous; Madagascar ... Solanum trichopetiolatum D’Arcy \& Rakot.

15a Leaf pubescence variable, not very sparse, along veins and lamina; flowers heterostylous; mountains of continental Africa

Solanum runsoriense C.H.Wright

16a Abaxial leaf surfaces with tufts of trichomes in the vein axils (domatia).....17

16b Abaxial leaf surfaces with trichomes on lamina and/or along veins, not with prominent tufts in the vein axils (domatia)

17a Inflorescence many times branched, open and with many flowers (more than 20); calyx lobes broadly deltate; petioles to $4 \mathrm{~cm}$ long, thin and flexuous; Mayotte (Comoros)

Solanum macrothyrsum Dammer

17b Inflorescence furcate, more congested and with fewer flowers (fewer than 20); calyx lobes deltate; petioles to $2.5 \mathrm{~cm}$ long, thicker; Madagascar...............18

18a Calyx lobes 0.8-2 mm long; inflorescences with 10-16 flowers

Solanum ivobibe D'Arcy \& Rakot.

18b Calyx lobes 4-6 mm long; inflorescences with 3-10 flowers

Solanum sambiranense D'Arcy \& Rakot.

19a Abaxial leaf surfaces evenly pubescent on veins and lamina

19b Abaxial leaf surfaces pubescent only along the veins and midrib, the trichomes not extending to the lamina.....

20a Anther pores lengthening to slits with age; flowers heterostylous; leaves evenly distributed along branches; mountains of continental Africa...

Solanum runsoriense C.H.Wright

20b Anther pores not lengthening to slit with age; flowers not heterostylous; leaves usually at least somewhat clustered on short shoots; Madagascar.....21

21a Leaves densely pubescent with golden (when dry) loosely dendritic trichomes; flowers more than $2 \mathrm{~cm}$ in diameter; anthers 4-6 mm long; widespread in Madagascar.

Solanum imamense Dunal

21b Leaves sparsely pubescent with white (when dry) congested dendritic trichomes; flowers $2 \mathrm{~cm}$ in diameter or less; anthers 3.5-4 mm long; dry forests of southern Madagascar..... Solanum betroka D’Arcy \& Rakot.

22a Inflorescence unbranched, with few flowers; pedicels $1.8-4.5 \mathrm{~cm}$ long Solanum humblotii Bitter

22b Inflorescence many times branched, with many flowers; pedicels $0.8-1.2 \mathrm{~cm}$ long

23a Anther pores lengthening to slits with age; flowers heterostylous; pedicels with pubescence like the inflorescence rhachis; mountains of continental Africa

Solanum runsoriense C.H.Wright

23b Anther pores not lengthening to slit with age; flowers not heterostylous; pedicels always glabrous; Madagascar.

Solanum madagascariense Dunal 


\section{Key 2. Normania CLADE (descriptions on Solanaceae Source)}

1a Leaves shallowly lobed, pubescent with long, tangled eglandular trichomes; anthers tapering, horned near the base, tightly connivent; seeds more than 5 mm long; fruit a dry berry; Mediterranean Solanum herculeum Bohs

$1 \mathrm{~b} \quad$ Leaves simple or ternate, glabrous or pubescent, but the trichomes not long and tangled, glandular; anthers markedly horned, spreading; seeds less than or equal to $5 \mathrm{~mm}$ long; fruit a brightly coloured, juicy berry; laurisylva forest

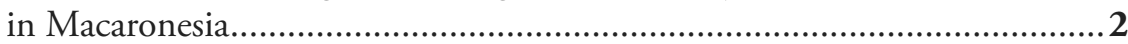

2a Leaves simple or ternate, the base truncate or cordate if leaves unlobed; anthers yellow, horned in lower third; berry bright red; Madeira

Solanum trisectum Dunal

2b Leaves simple, the base cordate; anthers black, horned about halfway up from the base; berry orange or red; Tenerife, Canary Islands

Solanum nava Webb \& Berthel.

Key 3. Dulcamaroid clade (descriptions in Knapp 2013 and on Solanaceae Source)

1a Buds turbinate and strongly pointed; petals strongly reflexed, with shiny green dots at the base of each; anthers tightly connivent with "glue"; fruit a shiny red berry, often ellipsoid; native plants in Mediterranean northern Africa

Solanum dulcamara L.

1b Buds rounded, often somewhat inflated; petals spreading, without shiny green dots; anthers not tightly connivent with "glue"; fruit red or black, globose; cultivated plants, occasionally naturalised throughout the region .......2

2a Flowers white; anthers on equal filaments; leaves with axillary tufts of trichomes on the lower surfaces (domatia), usually simple, occasionally pinnatifid; berry (very rarely) black.

Solanum laxum Spreng.

2b Flowers purple; one filament slightly longer than the other 4; leaves completely glabrous, pinnatifid, rarely simple; berry bright shiny red

Solanum seaforthianum Andrews

Key 4. MorelLoID CLAde (descriptions in Särkinen et al. 2018 and on Solanaceae Source)

1a Leaves shallowly to deeply pinnatifid ................... Solanum triflorum Nutt.

1b Leaves entire to sinuate-dentate ............................................................ 2

2a Glandular trichomes present (e.g. along stems, petioles and leaves), plants usually sticky to touch when fresh............................................................. 3

2b Glandular trichomes absent (e.g. along stems, petioles and leaves), plants not sticky to touch when fresh ........................................................................14

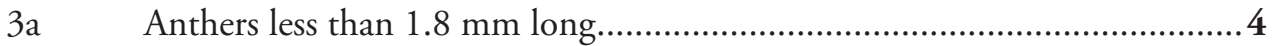


$3 \mathrm{~b} \quad$ Anthers more than or equal to $1.8 \mathrm{~mm}$ long......

4a Inflorescences with 10-40 flowers; pedicels spaced 1-2 mm apart, sharply bent at the base (near articulation point) in flower and fruit

Solanum tarderemotum Bitter

$4 b$

Inflorescences with 2-5(-10) flowers; pedicels spaced 0-1 mm apart, nodding, erect or spreading in flower and fruit, reflexed and slightly curved in some species in fruit but never in flower 5

$5 \mathrm{a}$

Calyx lobes $1-1.5 \mathrm{~mm}$ long in flower; fruiting calyces not accrescent, the tube remaining $1-1.7 \mathrm{~mm}$ long and lobes $1-1.5 \mathrm{~mm}$ long; fruit black when ripe, not markedly shiny, with a glaucous cast.....

Solanum retroflexum Dunal Calyx lobes $1.5-2.5 \mathrm{~mm}$ long in flower; fruiting calyces accrescent, the tube 3-4 mm long and lobes $2.5-8.0 \mathrm{~mm}$ long; fruit green when ripe, shiny ..... 6 Leaf bases attenuate to cuneate; inflorescences mostly intermodal, with 4-8(10) flowers; pedicels spaced $0.3-1 \mathrm{~mm}$ apart; calyx lobes $1.7-2.5 \mathrm{~mm}$ long; corollas with yellow-green central eye with black-purple V-shaped margins; anthers 1.0-1.4 mm long; berries dark green to green-brown marbled with white lines, becoming usually translucent and glossy, lower half of berries covered with enlarged calyces but berry mostly visible; seeds brown; stone cells (1-)2-3, these $0.5 \mathrm{~mm}$ in diameter; northern Africa

Solanum nitidibaccatum Bitter

$6 \mathrm{~b}$ Leaf bases truncate; inflorescences mostly leaf-opposed, with 2-5(-7) flowers; pedicels spaced $0(-1) \mathrm{mm}$ apart; calyx lobes $1.5-2.0 \mathrm{~mm}$ long; corolla with yellow-green or translucent basal star without black-purple colouration; anthers 1.2-2.0 mm long; berries pale green, shiny becoming dull, opaque, usually completely enveloped by enlarged calyces; seeds pale yellow; stone cells 4-6, these (0.5-)0.8-1 mm in diameter; only known from South Africa Solanum sarrachoides Sendtn.

Anthers more than or equal to $2.8 \mathrm{~mm}$ long 8

Anthers less than $2.8 \mathrm{~mm}$ long.

Inflorescences with bracteoles present in most individuals; buds narrowly ellipsoid; corolla deeply stellate, the lobes narrowly lanceolate; berries with more than 30 stone cells Solanum triflorum Nutt. Inflorescences never with bracteoles; buds globose, ovoid or narrowly ellipsoid; corolla rotate-stellate, the lobes long- triangular with rounded tips; berries with (0-)2-4 stone cells Solanum memphiticum Forssk.

9a Calyx lobes appressed to spreading in fruit, never strongly reflexed ............10

9b Calyx lobes strongly reflexed in fruit ................................................12

10a Calyx accrescent in fruit, calyx tube 3-4 mm long and lobes $2.5-8 \mathrm{~mm}$ long Solanum sarrachoides Sendtn.

10b Calyx not accrescent in fruit, calyx tube 1-2 mm long and lobes $1-1.5 \mathrm{~mm}$ long 
11a Buds ellipsoid; calyx tube 1.5-2.0 mm long, lobes 1-1.5 mm long, elongatedeltate with rounded tips; fruiting pedicels persist when fruits mature and fall off; Cameroon line (Cameroon and Equatorial Guinea), above 2,000 m elevation

Solanum pseudospinosum C.H.Wright

$11 \mathrm{~b}$ Buds subglobose; calyx tube $0.8-1.0 \mathrm{~mm}$ long, lobes $0.5-0.8 \mathrm{~mm}$ long, triangular with rounded to acute tips; fruiting pedicels generally do not persist and fall off with maturing fruits; in continental Africa only in South Africa and around the Mediterranean.

Solanum nigrum $\mathrm{L}$.

12a Leaves rhomboidal to lanceolate; filaments $1.2-1.5 \mathrm{~mm}$ long, anthers 1.3 1.8(-2) mm long; seeds $1.6-1.8 \mathrm{~mm}$ long and $1.3-1.5 \mathrm{~mm}$ wide.....

Solanum retroflexum Dunal

$12 \mathrm{~b}$ Leaves broadly to narrowly ovate to elliptic; filaments $0.5-1.3 \mathrm{~mm}$ long; anthers 1.8-2.5 mm long; seeds 1.8-2.2 mm long and 1.5-1.7 mm wide .... 13 13a Calyx with broad and relatively transparent sinuses, lobes elliptic to triangular, rounded at tip; free part of the filaments 1.0-1.3 mm long; mature berries slightly ellipsoid, shiny yellow, orange or red; stone cells always absent

Solanum villosum Mill.

13b Calyx with narrow, sharp triangular sinuses, lobes deltate with acute or rounded tips; free part of the filaments $0.5-0.7 \mathrm{~mm}$ long; mature berries round, dull black or green; stone cells 0-4; in Africa only in South Africa and around the Mediterranean Solanum nigrum L.

14a Anthers less than $1.8 \mathrm{~mm}$ long.................................................... 15

$14 \mathrm{~b} \quad$ Anthers more than or equal to $1.8 \mathrm{~mm}$ long .................................... 17

15a Pedicels spaced 1-2 mm apart, pedicels sharply bent at the base (near the articulation point) in flower and fruit Solanum tarderemotum Bitter

15b Pedicels spaced 0-0.5 mm apart, pedicels nodding, erect or spreading in flower and fruit....

16a Leaves with entire margins, occasionally sinuate-dentate; calyx lobes $0.3-0.5$ $\mathrm{mm}$ long in flower, 1(-2) $\mathrm{mm}$ in fruit; mature fruits black, the surface very shiny. Solanum americanum Mill.

$16 \mathrm{~b}$ Leaves shallowly toothed, occasionally entire; calyx lobes $1.0-1.5 \mathrm{~mm}$ long in flower, 1.5-2 mm in fruit; mature fruits purple-black or green, the surface dull. Solanum retroflexum Dunal

17a Anthers less than $2.8 \mathrm{~mm}$ long................................................ 18

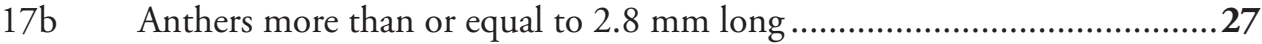

18a Berries without stone cells.............................................................. 19

18b Berries with 2-22 stone cells ...........................................................24

19a Pedicels persisting and not dropping with mature fruits; calyx lobes in fruit

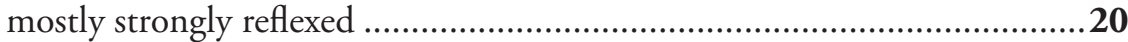

19b Pedicels dropping with mature fruits; calyx lobes in fruit appressed to slightly spreading, rarely strongly reflexed ............................................... 22 
20a Leaves rhomboidal to lanceolate; filaments $1.2-1.5 \mathrm{~mm}$ long, anthers 1.31.8(-2) $\mathrm{mm}$ long; seeds $1.6-1.8 \mathrm{~mm}$ long and $1.3-1.5 \mathrm{~mm}$ wide.

Solanum retroflexum Dunal

$20 \mathrm{~b}$ Leaves broadly to narrowly ovate to elliptic; filaments $0.5-1.3 \mathrm{~mm}$ long; anthers $1.8-2.5 \mathrm{~mm}$ long; seeds $1.8-2.2 \mathrm{~mm}$ long and $1.5-1.7 \mathrm{~mm}$ wide ....21

21a Calyx with broad and relatively transparent sinuses, lobes elliptic to triangular, rounded at tip; filaments $1.0-1.3 \mathrm{~mm}$ long; mature berries slightly ellipsoid, shiny yellow, orange or red; stone cells always absent

Solanum villosum Mill.

21b Calyx with narrow, sharp triangular sinuses, lobes deltate with acute tips; filaments $0.5-0.7 \mathrm{~mm}$ long; mature berries round, dull black or green; stone cells generally absent ( $2-4$ stone cells common in Asian material)

Solanum nigrum $\mathrm{L}$.

22a Buds elongate-oblong; fruiting peduncles strongly deflexed at the base (bent downwards at junction with the stem) .........Solanum chenopodioides Lam.

22b Buds ellipsoid to subglobose; fruiting peduncles straight or ascending ......23

23a Pedicels spaced 1-2 mm apart, sharply bent at the base (near the articulation point) in flower and fruit; seeds $1.5-2 \mathrm{~mm}$ long and $1-1.5 \mathrm{~mm}$ wide.

Solanum tarderemotum Bitter

23b Pedicels spaced $0-0.7 \mathrm{~mm}$ apart, straight, spreading or reflexed in flower and fruit; seeds $1.8-2 \mathrm{~mm}$ long and $1.5-1.6 \mathrm{~mm}$ wide....... Solanum nigrum $\mathrm{L}$.

24a Prostrate herb; leaves narrowly elliptic to lanceolate, base strongly attenuate; inflorescences with 1-5 flowers; pedicels stout and spreading; calyx lobes linear-oblong with rounded apices; mountains of Ethiopia

Solanum hirtulum C.H.Wright

24b Upright or spreading herb; leaves broadly ovate to elliptic, base acuminate, acute, obtuse, truncate to abruptly attenuate; inflorescences with 2-40 flowers; pedicels thinner, spreading to strongly reflexed; calyx lobes triangular, broadly deltoid or ovate with acute to rounded apices................................25

25a Pedicels strongly bent downwards at the base (near articulation point) in flower and fruit.....

Solanum tarderemotum Bitter

25b Pedicels spreading, stout or pendent in flower, occasionally recurved in fruit but never strongly bent downwards at the base .........................................26

26a Inflorescences unbranched or more often branched, often with small leaves (bracteoles?); calyx lobes broadly deltate to mere enations of the rim; style exserted 1.0-1.5 mm beyond anther cone; mature berries 3-4(-5) $\mathrm{mm}$ in diameter, dull yellowish brown

Solanum umalilaense Manoko

26b Inflorescences unbranched, never with small leaves; calyx lobes triangular; style exserted $0-1 \mathrm{~mm}$ beyond anther cone; mature berries $6-10 \mathrm{~mm}$ in diameter, dull black. Solanum nigrum $\mathrm{L}$.

27a Inflorescences with bracteoles present in most individuals; buds narrowly elliptic; berries with more than 30 stone cells Solanum triflorum Nutt.

$27 \mathrm{~b}$ Inflorescences never with bracteoles; buds globose, ovoid or narrowly ellipsoid; berries with $0-14$ stone cells 
28a Berries with 2-14 stone cells; leaf base strongly attenuate

Solanum hirtulum C.H.Wright

28b Berries without stone cells; leaf base not strongly attenuate.

29a Buds elongate-oblong; calyx lobes broadly deltate to triangular with acute tips; fruiting peduncles strongly bent at the base near junction with the stem; fruiting pedicels thin, reflexed and slightly recurved; seeds $1.2-1.4 \mathrm{~mm}$ long and $1.0-1.2 \mathrm{~mm}$ wide Solanum chenopodioides Lam.

29b Buds globose-subglobose; calyx lobes broadly deltate with rounded tips; fruiting peduncles straight; fruiting pedicels stout, erect and spreading; seeds 2-2.8 $\mathrm{mm}$ long and $1.5-1.8 \mathrm{~mm}$ wide

Solanum scabrum Mill.

\section{Key 5. Potato CLADe (descriptions on Solanaceae Source)}

1a Flowers yellow; anthers tightly connivent and tapering to a beak-like tip; fruit a bright red berry; cultivated tomatoes........................................................ 2

1b Flowers white or purple; anthers ellipsoid, not tapering to a beak-like tip; fruit green or whitish green (often with purple stripes)................................ 3

2a Corolla lobes deltate to triangular; anther cone stout, the style included; berry usually more than $1 \mathrm{~cm}$ in diameter (often much larger), fewer than 20 per infructescence; leaflets with serrate margins; cultivated tomato

Solanum lycopersicum L.

$2 \mathrm{~b} \quad$ Corolla lobes narrowly triangular; anther cone long and narrow, the style exserted; berry less than $1 \mathrm{~cm}$ in diameter, more than 20 per infructescence; leaflets with entire margins; cultivated currant tomato

Solanum pimpinellifolium $\mathrm{L}$.

3a Leaves at most ternate, usually simple; fruit a berry more than $3 \mathrm{~cm}$ in diameter; plant not bearing underground tubers; cultivated pepino

Solanum muricatum Aiton

3b Leaves pinnate; fruit a berry less than $3 \mathrm{~cm}$ in diameter; plant bearing underground tubers; cultivated potato

Solanum tuberosum $\mathrm{L}$.

Key 6. Brevantherum Clade (descriptions on Solanaceae Source)

1a Young flower buds turbinate; calyx densely pubescent within over entire surface; young stems sulcate; axillary leaves absent

Solanum erianthum D.Don

1b Young flower buds oblong to orbicular; calyx lobes pubescent within only in distal quarter; young stems terete; axillary leaves common 
Key 7. Leptostemonum Clade (descriptions in Vorontsova and Knapp 2016 and on Solanaceae Source)

1a Young stems and petioles noticeably winged; mature fruit densely pubescent; invasive plant in Tanzanian highlands and South Africa

Solanum robustum $\mathrm{H}$. Wendl.

$1 \mathrm{~b} \quad$ Young stems and petioles not markedly winged (terete or slightly ridged); mature fruit glabrous; native or invasive, widespread................................... 2

2a Flowers with stamens of differing lengths (due either to unequal anthers or unequal filaments or both); arid eastern and north-eastern Africa ................. 3

2b Flowers with all stamens equal in length; widespread .................................8

3a Corolla strongly zygomorphic, with the two lower lobes much larger; flowers often enantiostylous; Canary Islands ............................................................. 4

3b Corolla only weakly zygomorphic, the lower lobes somewhat but not markedly larger; flowers not enantiostylous; continental Africa ............................

4a Leaves narrowly elliptic to lanceolate; calyx lobes linear and awn-like; corolla always 5-merous; ripe berry orange; Gran Canaria... Solanum lidii Sunding

$4 \mathrm{~b} \quad$ Leaves ovate; calyx lobes linear; corolla often 4-merous; ripe berry yellow or yellowish green; Tenerife and Gran Canaria .........Solanum vespertilio Aiton

$5 \mathrm{a}$ Leaves orbicular to reniform, $1.2-2.5 \mathrm{~cm}$ long, wider than long; petioles longer than leaves; rare in north-eastern Somalia.

Solanum cymbalariifolium Chiov.

$5 b \quad$ Leaves ovate to elliptic or lanceolate, $2-14 \mathrm{~cm}$ long, longer than wide; petioles shorter than leaves; arid eastern and north-eastern Africa .....................6

6a Stem prickles dense, acicular, less than $0.5 \mathrm{~mm}$ wide at base, pale yellow; fruit fully concealed by the accrescent calyx .............. Solanum coagulans Forssk.

$6 \mathrm{~b}$ Stem prickles absent or sparse, if present wider than $1 \mathrm{~mm}$ at base, yellow to orange or brown; fruit at least partly exposed.............................................. 7

7a Leaves subentire to lobed; anthers of equal length; seeds very dark brown to almost black................................... Solanum melastomoides C.H.Wright

$7 \mathrm{~b}$ Leaves entire; one anther much longer than the others; seeds dull yellow to orange-brown Solanum somalense Franch.

8a Flower one per inflorescence, peduncle and rhachis absent; corolla pentagonal, lobed for $1 / 4-1 / 3$ of the way to the base, $0.9-1.3 \mathrm{~cm}$ in diameter; southern Africa

Solanum supinum Dunal

8b Flower usually more than one per inflorescence, peduncle and/or rhachis present in at least some inflorescences; corolla usually stellate, lobed for more than $1 / 3$ of the way to the base or, if lobed, for $1 / 4-1 / 3$ of the way to the base then corolla of long-styled flowers broader than $1.3 \mathrm{~cm}$ in diameter; widespread....9

9a Trichomes on young stems and adaxial (upper) surfaces of the leaves simple

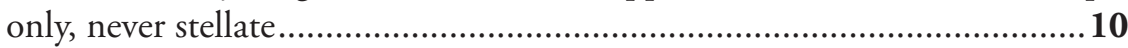

$9 \mathrm{~b}$ Trichomes on young stems and adaxial (upper) surfaces of the leaves stellate... 13 
10a Flowers pale bluish purple; anthers $8-12.5 \mathrm{~mm}$; fruit globose or extended into a "nipple" Solanum mammosum $\mathrm{L}$.

10b Flowers white or greenish white; anthers 5-7.5 mm; fruit globose ............11

11a No stellate hairs anywhere on the plant; fruit bright orange at maturity; seeds winged, 4-6 $\mathrm{mm}$ long Solanum capsicoides All.

11b Stellate hairs almost always present on the abaxial (lower) surface of the leaves; fruit yellow at maturity; seeds not winged, $1.8-2.8 \mathrm{~mm}$ long.....

12a Leaf lobes $2-3$ pairs, extending $1 / 3-1 / 2$ distance to the midvein; calyx lobes $5-6.5 \mathrm{~mm}$ long, often caudate. Solanum aculeatissimum Jacq.

12b Leaf lobes 3-5 pairs, extending less than $1 / 3$ of the distance to the midvein; calyx lobes $0.8-2 \mathrm{~mm}$ long, acute Solanum viarum Dunal

13a Leaves entire, 3-10 times longer than wide; shrubs erect, 1-6 m tall; stem trichomes with partly fused rays; southern Madagascar......

13b Leaves entire or lobed, 1-3(8) times longer than wide; shrubs erect, scandent or climbing, 0.2-6 m tall; if leaves entire and more than 3 times longer than wide, then shrub less than $1 \mathrm{~m}$ tall and not in southern Madagascar; stem trichomes with free rays.....

$14 \mathrm{a}$ Leaves 9-13(20) cm long; corolla $2-3.1 \mathrm{~cm}$ in diameter; juvenile branches with dark red prickles; south-eastern Madagascar.

Solanum croatii D'Arcy \& Keating

14b Leaves 1.5-7 cm long; corolla 1-2 cm in diameter; juvenile branches with grey-brown or red-brown prickles; south-western Madagascar ..... 15

15a Leaf blades 3-7 cm long, concolorous to weakly discolorous, yellow-green... Solanum bumeliifolium Dunal

15b Leaf blades 1.5-3(4) cm long, strongly discolorous, green-brown adaxially and glaucous abaxially..... Solanum heinianum D'Arcy \& Keating

16a Prickles and leaf venation noticeably dark orange to red, contrasting with the yellow-green to red-green leaf surface; southern Madagascar.....

Solanum pyracanthos Lam.

16b Prickles and leaf venation not a contrasting colour, yellow to green or redbrown; widespread or naturalised..............................................................17

17a Mature fruit green, never developing to bright yellow, orange or red; plants weakly andromonoecious; fruits $1-1.5 \mathrm{~cm}$ in diameter....

$17 \mathrm{~b}$ Mature fruit yellow, orange or red; plants hermaphroditic or andromonoecious, fruits $0.5-6 \mathrm{~cm}$ in diameter, if andromonoecious, then fruits more than $1.5 \mathrm{~cm}$ in diameter.

18a Shrub to tree 1.5-9 m tall; young stems and leaves densely ferruginous pubescent; trichomes on the inflorescences and pedicels not glandular; prickles straight to slightly curved.... Solanum chrysotrichum Schltdl.

$18 \mathrm{~b}$ Shrub to $3 \mathrm{~m}$; young stems and leaves pubescent green to brownish; trichomes on the inflorescences and pedicels glandular; prickles curved 
19a Mature fruit yellow or greenish yellow, 1.5-5(6) $\mathrm{cm}$ in diameter; corolla on long-styled flowers (1.3)2-6 cm in diameter; plants mostly andromonoecious

$19 \mathrm{~b}$ Mature fruit orange to red, $0.5-1.2(1.7) \mathrm{cm}$ in diameter; if mature fruit orange (1) $1.5-2.5(5) \mathrm{cm}$ in diameter, the plant cultivated; corolla on longstyled flowers $0.8-3 \mathrm{~cm}$ in diameter; plants mostly hermaphroditic

20a Cultivated tree 5-10 m tall with copious flowers; corolla bright purple aging to white with both colours usually present in an inflorescence, $5.5-8 \mathrm{~cm}$ in diameter Solanum wrightii Benth.

$20 \mathrm{~b}$ Wild plants or cultivated vegetables, less than $6 \mathrm{~m}$ tall; corolla white to mauve or purple with the colour constant within each individual, $1.6-6 \mathrm{~cm}$ in diameter

21a Petiole usually decurrent, leaf bases attenuate (cuneate); trichomes on abaxial leaf surface (if present) stalked with 4(5) rays............................................22

21b Petiole never decurrent, leaf bases cordate to cuneate; trichomes on abaxial leaf surface (if present) sessile or stalked with $6-16$ rays ............................23

22a Plant clearly stellate-pubescent and armed, drying yellow-green to red-brown; wild plant ............................. Solanum dasyphyllum Schumach, \& Thonn.

22b Plant usually glabrous and unarmed, drying a distinctive red-brown colour; cultivated plant. Solanum macrocarpon $\mathrm{L}$.

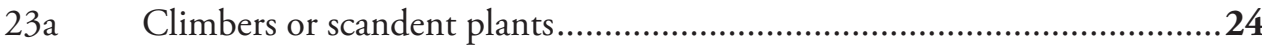

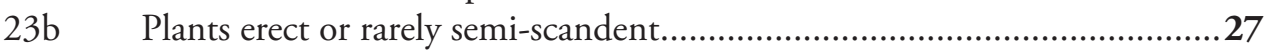

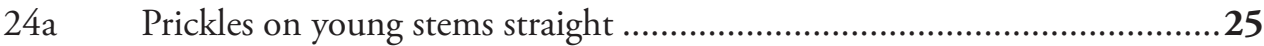

24b Prickles on young stems strongly curved .............................................26

25a Prickles pale straw-yellow, to $20 \mathrm{~mm}$ long; petiole with sessile stellate trichomes; corolla ca. $2.5 \mathrm{~cm}$ in diameter; style strongly curved; Mediterranean northern Africa (adventive from Asia).... Solanum virginianum $\mathrm{L}$.

25b Prickles yellow (but not straw-coloured) or brown; petiole trichomes usually stalked; corolla 3.5-5 cm in diameter; style straight; southern Kenya.

Solanum nigriviolaceum Bitter

26a Corolla white, $1.3-1.6 \mathrm{~cm}$ in diameter; seeds 5.5-6 mm long; Kenyan mountains Solanum agnewiorum Voronts.

26b Corolla mauve to purple, $3.5-6 \mathrm{~cm}$ in diameter; seeds 3-4 mm long; eastern and southern Africa, Madagascar Solanum richardii Dunal

27a Calyx inflated, fully covering the berry at maturity; young stems densely covered with straight prickles; northern Madagascar

Solanum mahoriense D'Arcy \& Rakot.

27b Calyx not inflated, the berry exposed at maturity; young stems prickly or unarmed; widespread.....

28a Corolla lobed for more than half way to the base; shrubs or trees up to $6 \mathrm{~m}$; variety of habitats.

28b Corolla lobed for half way to the base or less; small shrubs up to $2 \mathrm{~m}$; weeds of open arid environments or cultivated crops; relatives of the eggplant ....35 
29a Leaves 8-18 cm long, strongly discolorous; young fruits plain green; seeds $3.5-4.5 \mathrm{~mm}$ long; wet upland habitats.

29a Leaves 2-8 cm long, concolorous or sometimes discolorous; young fruits striped in different shades of green; seeds $2.2-3.5 \mathrm{~mm}$ long; arid upland or lowland habitats.

30a Fruit globose, never apiculate, $1.4-1.7 \mathrm{~cm}$ in diameter, 4-10 per infructescence; young stems with yellow (when dry) long-stalked trichomes, the stalks $1-3 \mathrm{~mm}$

Solanum thomsonii C.H.Wright

$30 \mathrm{~b}$ Fruit turbinate or cone-shaped, sometimes globose, usually somewhat apiculate, $2.8-5 \times 1.8-4.5 \mathrm{~cm}, 1-3(5)$ per infructescence; young stems usually lacking long-stalked yellow (when dry) trichomes.

31a Fruit distinctly turbinate or cone-shaped, $2.8-3.7 \times 1.8-2.2 \mathrm{~cm}$; leaves on fertile branches elliptic and subentire, $6-8 \times 2.5-4 \mathrm{~cm}$, ca. 2.5 times longer than wide; $2100-3000$ m elevation

Solanum phoxocarpum Voronts.

31b Fruit globose, usually somewhat apiculate, 3-5 x 2-4.5 cm; leaves on fertile branches ovate(elliptic) and lobed(subentire), 8-15 x 6-12 cm, 1.5-2 times longer than wide; $1200-2100(3200)$ m elevation

Solanum aculeastrum Dunal

32a Prickles straight or occasionally curved; petioles $1 / 3-2 / 3$ as long as the leaf blades

Solanum polbillii Voronts.

32b Prickles on young stems strongly curved; petioles less than $1 / 3$ of the leaf

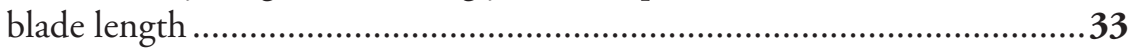

33a Leaves entire, densely stellate-pubescent on both sides; eastern and northeastern African highlands.

Solanum dennekense Dammer

33b Leaves lobed, adaxially glabrescent; eastern and north-eastern Africa .........34

34a Leaves 2-4 cm long; curved prickles on young stems 5-10 mm long; eastern and north-eastern Africa

Solanum arundo Matthei

34b Leaves 6-8 cm long; curved prickles on young stems 1-3 mm long; coastal Kenya, rare Solanum malindiense Voronts.

35a Fruit with soft pericarp, in a variety of shapes and colours, edible; common fasciation in the flowers (e.g. increase in the number of flower parts up to 8), inflated ovaries; cultivated species Solanum melongena $\mathrm{L}$.

35a Fruit spherical, yellow, with comparatively hard pericarp, not edible; flowers 5-merous; wild plants 36

36a Leaves lobed with primary and secondary lobes, the primary lobes extending 2/3-3/4 of the distance to the midvein and secondary lobes always present; southern Africa and northern African coasts around the Mediterranean Solanum linnaeanum Hepper \& P.M-L.Jaeger

$36 \mathrm{~b}$ Leaves entire or lobed, lobes extending up to $2 / 3$ of the distance to the midvein, secondary lobes usually not present; widespread 
37a Leaf margins and venation nearly white and contrasting with greenish redbrown adaxial leaf surface; trichomes on the abaxial surface of the leaves with 10-17 rays; Ethiopian highlands Solanum marginatum L.f.

37b Leaf margins and venation the same colour as the rest of the leaf blade; trichomes on the abaxial surface of the leaves with 5-12(15) rays; widespread.

38a Leaf lobes apically obtuse to acute, sometimes rounded, sometimes with secondary lobes; lobes $1 / 4-2 / 3$ of the distance to the midvein; leaves and young stems glabrescent to moderately pubescent

38b Leaf lobes apically rounded, sometimes obtuse, never with secondary lobes; lobes up to $1 / 3(1 / 2)$ of the distance to the midvein; leaves and young stems usually densely pubescent

39a Calyx lobes on long-styled flowers 7-10 mm long, ovate and foliaceous, apically obtuse; South Africa. Solanum umtuma Voronts. \& S.Knapp

39b Calyx lobes on long-styled flowers 4-7 mm long, deltate or long-triangular apically acuminate; northern Africa and Cape Verde Islands

40a Calyx lobes on long-styled flowers $4-7 \mathrm{~mm}$ long, deltate, ca. 1/6 as long as the fruit at maturity; continental Africa north of the equator.

Solanum cerasiferum Dunal

40b Calyx lobes on long-styled flowers 6-7 mm long, long triangular, $1 / 2$ to $1 / 3$ as long as the fruit at maturity; Cape Verde Islands (Senegal?)

Solanum rigidum Lam.

41a Prickles straight; corolla on long-styled flowers $1.8-2.5 \mathrm{~cm}$ in diameter; anthers ca. $4.5 \mathrm{~mm}$ long; Madagascar; Mauritius, Réunion

Solanum insanum L.

$41 \mathrm{~b}$ Prickles curved or straight; corolla on long-styled flowers $2.5-4.5 \mathrm{~cm}$ in diameter; anthers 5-9 mm long; widespread....

42a Leaves usually entire, sometimes lobed; trichomes on the abaxial leaf surface sessile or with stalks up to $0.1 \mathrm{~mm}$ long; fruits $1.5-3 \mathrm{~cm}$ diameter

Solanum campylacanthum Hochst. ex A.Rich.

42b Leaves lobed; trichomes on the abaxial leaf surface with stalks up to 0.5(1) $\mathrm{mm}$ long; fruits $2.5-4.5 \mathrm{~cm}$ diameter .....

43a Leaves velvety red-brown adaxially; calyx lobes on long-styled flowers ovate to oblong, foliaceous, 7-10 $\mathrm{mm}$ long.......... Solanum aureitomentosum Bitter

43b Leaves yellow-green to green-brown adaxially; calyx lobes on long-styled flowers deltate, usually not foliaceous, $2.5-6 \mathrm{~mm}$ long...............................44

$44 \mathrm{a}$ Leaves concolorous to weakly discolorous, indumentum yellowish; leaves ca. 1.5 times longer than wide; young stems terete to angular; north-eastern Africa Solanum incanum $\mathrm{L}$.

$44 \mathrm{~b} \quad$ Leaves strongly discolorous, indumentum dirty greenish brown adaxially and whitish yellow abaxially; leaves 1.5-2.5 times longer than wide; young stems with somewhat raised longitudinal ridges; southern Africa. 
45a Leaves on fertile branches with distinct lobes, at least some of the lobes longer than $1 / 4$ of the distance from the midvein to the leaf edge. 46

45b Leaves on fertile branches entire or subentire or with some shallow lobes no longer than $1 / 4$ of the distance from the midvein to the leaf edge ............89

46a Leaves more than 3 times longer than wide........................................47

46b Leaves 1-3 times longer than wide..................................................49

47a Plant unarmed or prickles straight, orange to red; trichomes on the abaxial surfaces of the leaves with (9)12-14 rays....... Solanum elaeagnifolium Cav.

47b Plant densely armed with curved broad-based pale-yellow prickles; trichomes on the abaxial surfaces of the leaves, if present, with $0-8$ rays

48a Anthers 2.5-3.2 mm long; trichomes anywhere on the plant with 0-4 rays; South Africa

Solanum sodomaeodes Kuntze

48b Anthers 5-6.5 mm long; trichomes anywhere on the plant with 5-8 rays; north-eastern Africa.

Solanum glabratum Dunal

49a Leaves with at least some secondary lobing present ............................50

49b Leaves lobed once only .........................................................51

50a Flowers 3-7 per inflorescence, mauve to purple; anthers 5.5-7 $\mathrm{mm}$ long; South Africa................................................. Solanum rubetorum Dunal

50b Flowers 6-50 per inflorescence, white; anthers 9-10 $\mathrm{mm}$ long; invasive in South Africa and Kenya .......................... Solanum sisymbriifolium Lam.

51a No prickles visible anywhere on the plant .....................................52

51b At least some prickles visible on the plant .....................................57

52a Plant scandent or scrambling, sometimes erect; anthers (4)5-6.5 mm long; inland eastern Africa ...................... Solanum cyaneopurpureum De Wild.

52b Plant erect; anthers 2.3-5(8.5) mm long; widespread...........................53

53a Corolla $1.5-3 \mathrm{~cm}$ in diameter; mauve or purple; southern Africa, Indian Ocean islands..............................................................................54

53b Corolla $0.9-1.5 \mathrm{~cm}$ in diameter; white (only occasionally pale violet); widespread on continent or cultivated .................................................55

54a Fruiting pedicels deflexed, curved; anthers 3.5-5.2 mm long; South Africa and Namibia ..................................................Solanum burchellii Dunal

54b Fruiting pedicels straight and spreading; anthers 4.5-8.5 mm long; Mauritius and Réunion....

Solanum violaceum Ortega

55a Inflorescences 2.5-6 cm long, with 5-22 flowers ..... Solanum anguivi Lam.

55b Inflorescences $1-2.5 \mathrm{~cm}$ long, with $1-4(10)$ flowers................................56

56a Leaf blades 5-18 cm long, petioles 1-4 cm long; plant cultivated for leaves or fruits, widespread ......................................... Solanum aethiopicum L.

$56 \mathrm{~b}$ Leaf blades 3-8 cm long, petioles $0.5-1.5 \mathrm{~cm}$ long; wild plant in southern Africa

Solanum catombelense Peyr.

57a Prickles on young stems predominantly curved..................................58

57b Prickles on young stems predominantly straight, sometimes straight and reflexed ........................................................................70 
58a Young prickles and indumentum red; scandent shrubs; Madagascar

58b Young prickles and indumentum yellowish, green or brown; erect or scandent shrubs; widespread (mainland Africa, rarely Madagascar, Indian Ocean Islands)

59a Young stems with a dense covering of long-stalked trichomes and prickles of different lengths; 1000-3000 m elevation....Solanum myoxotrichum Baker

$59 \mathrm{~b} \quad Y o u n g$ stems with a sparse or dense covering of sessile or short-stalked trichomes of uniform lengths; 0-1000 m elevation

Solanum erythracanthum Dunal

60a Stem trichomes with stalks 0.1-0.4 mm long; anthers 6-10 mm long; coastal eastern Africa

Solanum usaramense Dammer

60b Stem trichomes sessile or the stalks to $0.1 \mathrm{~mm}$ long; anthers $3-8.5 \mathrm{~mm}$ long; widespread

61a Plants of the Indian Ocean islands (Mauritius, Réunion, Aldabra)...........62

61b Plants of continental Africa..........................................................63

62a Fruiting pedicels straight and spreading; corolla to $3 \mathrm{~cm}$ in diameter; petiole not winged from decurrent leaf base; Mauritius and Réunion

Solanum violaceum Ortega

62b Fruiting pedicels curved and deflexed; corolla to $2 \mathrm{~cm}$ in diameter; petiole slightly winged from decurrent leaf base; Aldabra (Seychelles)

Solanum aldabrense C.H.Wright

63a Calyx in flower 7-9 mm long, apically caudate; calyx in fruit 10-12 mm long; trichomes with midpoints much longer that the rays; Tanzanian highlands, rare

Solanum inaequiradians Bitter

63b Calyx in flower 2-6 mm long, apically acute to acuminate or rarely caudate; calyx in fruit less than $10 \mathrm{~mm}$ long; trichomes with the midpoints short than or equal to the rays; widespread

$64 \mathrm{a}$ Plant always erect; leaves discolorous; corolla $0.8-1.5 \mathrm{~cm}$ in diameter, lobed for $1 / 2-2 / 3(3 / 4)$ of the way to the base.

$64 \mathrm{~b} \quad$ Plant often scandent or semi-scandent; leaves usually concolorous; corolla (1) $1.5-2.5 \mathrm{~cm}$ in diameter, lobed for $2 / 3$ of the way to the base or more.. 66

65b Inflorescences 2.5-6 cm long, with 5-22 flowers; polymorphic weed across highland areas of Africa and Madagascar....

Solanum anguivi Lam.

65a Inflorescences 1-2.5 cm long, with 1-4 flowers; southern Africa

Solanum catombelense Peyr.

66a Prickles 3-5 mm long; leaf blades 1.5-2.5(9) cm long, leaf base narrow-cuneate to attenuate; South Africa and Namibia.

Solanum capense $\mathrm{L}$.

66b Prickles 1-4(7) $\mathrm{mm}$ long. Leaf blades (1)2.5-14 cm long, leaf base cuneate to truncate; widespread

67a Calyx 2-4 mm long in flower .......................................................68

67b Calyx 4-6 mm long in flower .....................................................69 
68a Prickles on young stems 1.5-2(3) mm long; leaves $2.5-5.5 \mathrm{~cm}$ long; seeds 2.5$3.5 \mathrm{~mm}$ long; inland eastern Africa ..... Solanum cyaneopurpureum De Wild.

68b Prickles on young stems 2-4 mm long. Leaves 3-14 cm long; seeds 1.8-2.5 mm long; coastal eastern Africa................... Solanum zanzibarense Vatke

69a Leaves drying yellow-green; petiole 1/4-1/3 of the leaf length; eastern and north-eastern Africa .................... Solanum hastifolium Hochst. ex Dunal

69b Leaves drying dark red-brown; petiole 1/3-1/2 of the leaf length; southern Africa Solanum torreanum A.E.Gonç.

70a Inflorescence with more than 10 flowers ........................................... 71

70b Inflorescence with 10 or fewer flowers...............................................74

71a Leaves 1-4(6) cm long; corolla 1.3-2.4 cm in diameter; seeds almost black; north of the equator.........................................Solanum forskalii Dunal

$71 \mathrm{~b}$ Leaves (5)7-25 cm long; corolla $0.7-1.5 \mathrm{~cm}$ in diameter; seeds yellow to orange-brown; widespread ...................................................... 72

72a Inflorescences $1-2.5 \mathrm{~cm}$ long, peduncle $0-2 \mathrm{~mm}$ long; trichomes on the young stems with (5)8-20 rays; western Africa Solanum anomalum Thonn.

72b Inflorescences 2.5-8 cm long, peduncle 2-30 mm long; trichomes on the young stems with 6-8 rays; widespread.

73a Inflorescences branched many times (more than once); Kenya and Tanzania

Solanum usambarense Bitter

$73 \mathrm{~b}$ Inflorescences unbranched or forked; widespread.... Solanum anguivi Lam.

74a Young prickles almost filiform and easily bendable like bristles, with no firm prickles anywhere on the plant; eastern African savannah

$74 \mathrm{~b} \quad$ Young prickles sturdy when touched; widespread

Solanum setaceum Dammer

$75 \mathrm{a}$

Calyx lobes oblong, foliaceous, apically obtuse; lowland eastern Africa, rare..

Solanum lamprocarpum Bitter

75b Calyx lobes deltate, sometimes long-ovate or narrow-oblong, not clearly foliaceous, apically acute to acuminate; widespread ...............................76

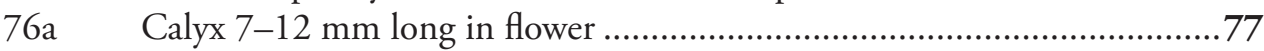

76b Calyx 2-7 mm long in flower ..................................................8 80

77a Prickles on young branches 8-16 mm long; leaves elliptic; Ethiopian highlands ................................................ Solanum macracanthum A.Rich.

77b Prickles on young branches 2-7 mm long; leaves ovate; Tanzania, South Africa and Madagascar..........................................................78

78a Corolla lobed for 3/4 of the way to the base or more; trichomes on the young stems with midpoints (0.5)1.5-2.5(3) mm long; Tanzanian highlands.

Solanum inaequiradians Bitter

$78 \mathrm{~b}$ Corolla lobed for $1 / 2-2 / 3$ of the way to the base; trichomes on the young stems with midpoints 0.15-5 mm long; South Africa and Madagascar.....79

79a Young stems with a dense covering of long-stalked trichomes and prickles of different lengths; Madagascar...................... Solanum myoxotrichum Baker

$79 \mathrm{~b} \quad$ Young stems with no bristles, only prickles and trichomes of uniform length; South Africa. Solanum tomentosum $\mathrm{L}$. 
80a Young prickles and indumentum red; pedicels (1)1.5-4 cm long; Madagascar ......

$80 \mathrm{~b}$ Young prickles and indumentum pale yellow to brown or purple, never red; pedicels $0.2-1(1.4) \mathrm{cm}$ long; mainland Africa, rarely in Madagascar .........82

81a Young stems with a dense covering of long-stalked trichomes and prickles of different lengths; 1000-3000 m elevation....Solanum myoxotrichum Baker

81b Young stems with a sparse or dense covering of sessile or short-stalked trichomes of uniform lengths; $0-1000$ m elevation

Solanum erythracanthum Dunal

82a Corolla lobed for $3 / 4$ of the way to the base or deeper; plants scrambling or climbing, sometimes erect....

82a Corolla lobed for $1 / 2-2 / 3$ of the way to the base; plants erect, sometimes scandent

83a Prickles on young stems 2-4 mm long; eastern African coastal areas

Solanum zanzibarense Vatke

83b Prickles on young stems (3)4-13 mm long; arid environments across continental Africa

84a Prickles reflexed; seeds nearly black .......................Solanum forskalii Dunal

84b Prickles perpendicular to the stem; seeds pale yellow to orange-brown ......85

85a Leaves 1-2 times longer than wide; leaf lobes to $2 / 3$ of the distance to the midvein; leaves concolorous to strongly discolorous; north-eastern Africa.....

Solanum adoense Hochst. ex A. Rich.

85b Leaves 1.5-3 times longer than wide; leaf lobes to $1 / 2$ of the distance to the midvein; leaves concolorous; South Africa

Solanum humile Dunal

86a Petiole $1 / 3-2 / 3$ as long as the leaf blade; South Africa

Solanum tomentosum $\mathrm{L}$.

86b Petiole $1 / 6-1 / 3$ as long as the leaf blade; widespread ................................87

87a Leaf blades (5)11-25 cm long; inflorescence with 5-22 flowers; common variable highland weed widespread across Africa ......Solanum anguivi Lam.

87b Leaf blades 1.7-9 cm long; inflorescence with 1-6 flowers; southern Africa..

88

88a Leaves elliptic; corolla $1.5-2.2 \mathrm{~cm}$ in diameter, mauve to purple

Solanum burchellii Dunal

88b Leaves ovate, sometimes oblong; corolla $0.9-1.3 \mathrm{~cm}$ in diameter, usually white

89a At least some inflorescences branched several to many times; inflorescence with (10)20-150 flowers; erect shrubs to small trees

89b Inflorescences unbranched or forked; inflorescence with 1-10(20) flowers; scrambling, scandent or erect shrubs ......................................................98

90a Plants with at least some visible prickles or bristles.................................91

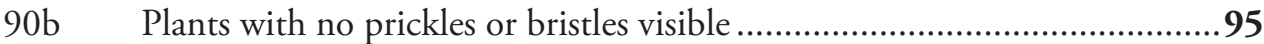

91a Young stems with soft or thin bristles...................................................92

91b Young stems with firm deltate prickles ..................................................93 
92a Stem bristles soft and white, 0.4-0.7 mm wide at base; leaves ca. 2 times longer than wide; 1500 -1800 m elevation; Tanzania

Solanum schliebenii Werderm.

92b Stem bristles erect purple-black or brown, 0.2-0.4 mm wide at base; leaves 2.5-3.5 times longer than wide; 1800-2600 m elevation; eastern Africa

Solanum schumannianum Dammer

93a Leaf blades 12-40 cm long, whitish grey underneath with trichomes, falling as white powder when touched; inflorescences with 30-150 flowers; anthers 2.5-3 mm long. Solanum giganteum Jacq.

93b Leaf blades 4-10(16) cm long, yellow-green, brown or rarely whitish grey underneath, trichomes not falling when touched; inflorescences with 10-30 flowers; anthers 3.5-6 mm long.

94

94a Leaf base cuneate to acuminate; anthers 3.5-4 mm long; stem trichomes with midpoints 0.4-1(2) mm long; Democratic Republic of the Congo, Rwanda, Uganda, Tanzania; rare.

Solanum wittei Robyns

$94 \mathrm{~b}$ Leaf base rounded, rarely cuneate; anthers 4-6 $\mathrm{mm}$ long; stem trichomes with midpoints 0.05-0.2 mm long; widespread. Solanum tettense Klotzsch

95a Leaves elliptic, with 8-12 pairs of primary veins, 2.5-3.5 times longer than wide

Solanum schumannianum Dammer

95b Leaves ovate, sometimes elliptic, with 4-8 pairs of primary veins, 1.5-2.5(3) times longer than wide............................................................96

96a Leaves concolorous; calyx lobes 2-4(5) mm long, long-acuminate; Sudan, Eritrea, Ethiopia, Somalia, 1500-2800 m elevation

Solanum schimperianum Hochst. ex A.Rich.

$96 \mathrm{~b}$ Leaves discolorous; calyx lobes 1-2 mm long, obtuse to acute, sometimes acuminate; southern Ethiopia, Somalia and further south, 650-1900 m elevation ...........................................................................997

97a Leaf base cuneate to acuminate; anthers 3.5-4 mm long; stem trichomes with midpoints 0.4-1(2) mm long; Democratic Republic of the Congo, Rwanda, Uganda, Tanzania; rare.

Solanum wittei Robyns

97b Leaf base rounded, rarely cuneate; anthers 4-6 $\mathrm{mm}$ long; stem trichomes with midpoints $0.05-0.2 \mathrm{~mm}$ long; widespread, common

Solanum tettense Klotzsch

98a Plants with no prickles visible ...................................................99

98b Plants with at least some visible prickles. .........................................109

99a Leaves 3-8 times longer than wide, narrow-elliptic or lanceolate, rarely nar-

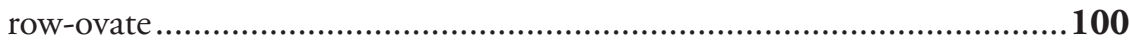

99b Leaves 1-3 times longer than wide, orbicular, ovate, obovate, elliptic or oblong 
100a Corolla $0.9-1.5 \mathrm{~cm}$ in diameter; trichomes on young stems with ca. 8 rays; native plant in eastern and north-eastern Africa, black cotton soils

Solanum lanzae J.-P.LeBrun \& Stork

100b Corolla 2.5-3(5) cm in diameter; trichomes on young stems with 10-14(16) rays; weed in southern Africa and Mediterranean....

Solanum elaeagnifolium Cav.

101a Leaves less than 1.5 times longer than wide, orbicular, ovate or obovate; arid north-eastern Africa....

102

101b Leaves more than 1.5 times longer than wide, ovate, elliptic or oblong; widespread

103

102a Erect shrub to small tree 1.5-4 m tall; leaves and flowers readily deciduous, so stems often bare; seeds orange-brown Solanum jubae Bitter

102a Scandent to erect shrub up to $1 \mathrm{~m}$ tall; leaves and flowers not readily deciduous, stems not bare; seeds dark brown to almost black

Solanum cordatum Forssk.

103a Leaf base attenuate; corolla lobed for ca. 3/4 of the way to the base; trichomes on the young stems with 10-20 rays.

103a Leaf base cuneate, rounded or truncate, rarely attenuate; corolla lobed for $1 / 2-2 / 3$ of the way to the base; trichomes on the young stems with $6-9(12)$ rays.....

104a Petioles $1 / 3-2 / 3$ as long as the adult leaves; flowers 4-8 per inflorescence; seeds 2.8-3.5 mm long; Angola

Solanum pauperum C.H.Wright

$104 \mathrm{~b}$ Petioles $1 / 6-1 / 4$ as long as the adult leaves; flowers $8-15$ per inflorescence; seeds 3.6-4.8 mm long; eastern and south-eastern Africa

Solanum goetzei Dammer

105a Calyx lobes 5-10 mm long in flower, foliaceous, ovate to elliptic; leaf apex rounded, sometimes obtuse; arid north-eastern Africa.

Solanum pampaninii Chiov.

105b Calyx lobes 1-4.5 mm long in flower, not clearly foliaceous, deltate; leaf apex acute or obtuse, sometimes rounded; widespread 106

106a Scandent shrub; inflorescences 3.5-7 cm long; young stems with stalked trichomes, the stalks $0.1-0.4 \mathrm{~mm}$ long; eastern African highlands.

Solanum stipitatostellatum Dammer

106b Erect herb or shrub; inflorescences 1-3.5 cm long; young stems with sessile or stalked trichomes, the stalks up to $0.15 \mathrm{~mm}$ long; widespread.... 107

$107 \mathrm{a}$ Fruit (1)1.5-2.5(5) cm in diameter; plant cultivated for leaves or fruits; widespread and common in western and eastern Africa

Solanum aethiopicum L.

107b Fruit (0.7)0.75-1.1 cm in diameter; wild plant in southern Africa .........108

108a Leaves elliptic; corolla $1.5-2.2 \mathrm{~cm}$ in diameter, mauve to purple.

Solanum burchellii Dunal

108b Leaves ovate, sometimes oblong; corolla $0.9-1.3 \mathrm{~cm}$ in diameter, usually white

Solanum catombelense Peyr. 
109a Prickles on young stems predominantly curved

109b Prickles on young stems predominantly straight, sometimes straight and reflexed

110a Most leaves on reproductive branches less than two times longer than wide..

110 b Most leaves on reproductive branches two times longer than wide or longer.

111a Corolla lobed for ca. 3/4 of the way to the base or deeper; seeds dark brown to almost black; continental Africa north of the equator

$111 \mathrm{~b}$ Corolla lobed for ca. $2 / 3$ of the way to the base; seeds yellow to orangebrown; continental Africa south of the equator.

112a Inflorescences with 1(2) flowers; trichomes on young stem with 12-18 rays. Solanum cordatum Forssk.

112b Inflorescences with (1)2-20 flowers; trichomes on young stem with 6-10 rays

Solanum forskalii Dunal

113a Leaves drying yellowish green; anthers 3-4 mm long; southern Africa

Solanum litoraneum A.E.Gonç.

$113 \mathrm{~b}$ Leaves drying reddish brown; anthers (4.5)5.5-8 mm long; Madagascar 114

$114 \mathrm{a}$ Young stems with a dense covering of long-stalked trichomes and prickles of different lengths; 1000-3000 m elevation

Solanum myoxotrichum Baker

$114 \mathrm{~b}$ Young stems with a sparse or dense covering of sessile or short-stalked trichomes of uniform lengths; $0-1000$ m elevation

Solanum erythracanthum Dunal

115a Plant erect, $0.4-1.5 \mathrm{~m}$ tall; corolla $0.9-1.4 \mathrm{~cm}$ in diameter; anthers $2.5-3.5$

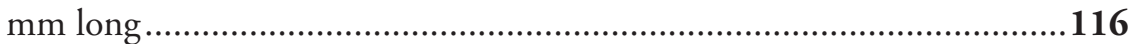

115b Plant scandent, sometimes erect; corolla (1.2) $1.5-3 \mathrm{~cm}$ in diameter; anthers 4-8 mm long......

116a Inflorescence 3-4(6) cm long; trichomes on abaxial surfaces of the leaves with stalks $0.15-0.4 \mathrm{~mm}$ long; eastern Africa. Solanum mauense Bitter

116b Inflorescence 1-2.5 cm long; trichomes on abaxial surfaces of the leaves with stalks $0-0.15 \mathrm{~mm}$ long; southern Africa. Solanum catombelense Peyr.

117a Calyx 7-9 mm long at anthesis, the lobes caudate; trichomes on young stems with midpoints (0.5)1.5-2.5(3) mm long; Tanzanian highlands

Solanum inaequiradians Bitter

117b Calyx 2-6 mm long at anthesis, the lobes acute to acuminate; trichomes on young stems with midpoints $0-1.5 \mathrm{~mm}$ long; widespread.

118

118a Leaves 3-6 times longer than wide, the bases attenuate; arid eastern and north-eastern Africa

Solanum glabratum Dunal

118b Leaves 2-3 times longer than wide, the bases cuneate to cordate; eastern Africa and Madagascar.

119a Corolla (1.8)2-3 cm in diameter; anthers (5.5)6-10 mm long ...............120

$119 \mathrm{~b}$ Corolla $1-2(2.4) \mathrm{cm}$ in diameter; anthers $4-6(7) \mathrm{mm}$ long 
120a Inflorescences with 1-2(5) flowers; young stems with trichomes sessile or stalked, the stalks up to $0.2 \mathrm{~mm}$ long; Madagascar.

Solanum erythracanthum Dunal

120b Inflorescences with 3-10 flowers; young stems with trichomes always stalked, the stalks $0.1-0.4 \mathrm{~mm}$ long; eastern Africa

121a Leaves 3-8 cm long, densely stellate-pubescent on both sides; eastern African coasts Solanum usaramense Dammer

121b Leaves 6-13 cm long, adaxially glabrescent; eastern African mountains Solanum stipitatostellatum Dammer

122a Leaves apically rounded, with thick-stalked trichomes on the adaxial surface; inflorescence with 1-2(3) flowers; eastern Africa..... Solanum taitense Vatke 122a Leaves apically acute to obtuse, sometimes rounded, without thick-stalked trichomes on the adaxial surface; inflorescence with (1)2-20 flowers ......123

123a Leaves with 2-3 pairs of primary veins; seeds dark brown to almost black; continental Africa north of the equator.

Solanum forskalii Dunal

123b Leaves with 4-6 pairs of primary veins; seeds yellow to orange-brown; continental Africa south of the equator or near the equator ............................124

$124 \mathrm{a}$ Prickles usually under $2 \mathrm{~mm}$ long; leaves $2.5-5.5 \mathrm{~cm}$ long; trichomes on the leaves and stems with stalks ca. $0.1 \mathrm{~mm}$ long; inland eastern Africa

Solanum cyaneopurpureum De Wild.

124b Prickles over $2 \mathrm{~mm}$ long; leaves 3-14 cm long; trichomes on the leaves and stems sessile or with stalks under $0.1 \mathrm{~mm}$ long; coastal eastern Africa

Solanum zanzibarense Vatke

125a Leaves more than two times longer than wide.......................................126

125b Leaves less than two times longer than wide.......................................130

126a Corolla 2.5-3(5) $\mathrm{cm}$ in diameter; anthers 6-10 $\mathrm{mm}$ long; invasive in southern Africa and around the Mediterranean ..... Solanum elaeagnifolium Cav.

126b Corolla 0.9-2.3 cm in diameter; anthers 2.5-6 mm long; Ethiopia, Tanzania or further south

127a Prickles on young stems $8-16 \mathrm{~mm}$ long; calyx $7-12 \mathrm{~mm}$ long at anthesis; Ethiopia................................................. Solanum macracanthum A.Rich.

127b Prickles on young stems 1-6 mm long; calyx 2.5-7 mm long at anthesis; Tanzania or further south.

128

128a Plant scrambling shrub; leaves almost glabrous, trichomes on the leaves with up to 4 rays; Tanzanian coastal forest, rare (possibly extinct)

Solanum ruvu Voronts.

128b Plant erect; leaves stellate-pubescent, trichomes on the abaxial sides of the leaves with $17-15$ rays; southern Africa

129a Leaves elliptic; corolla $1.5-2.2 \mathrm{~cm}$ in diameter, mauve to purple

Solanum burchellii Dunal

129b Leaves ovate, sometimes oblong; corolla $0.9-1.3 \mathrm{~cm}$ in diameter, usually white

Solanum catombelense Peyr. 
130a Leaves on reproductive branches less than $2 \mathrm{~cm}$ long ..............................131

130b Leaves on reproductive branches more than $2 \mathrm{~cm}$ long ..........................134

131a Leaves and flowers predominantly on short shoots, few on main stems; corolla lobed for $1 / 2-2 / 3$ of the way to the base; seeds yellow to orange-brown; southern Madagascar .....

131b Leaves and flowers predominantly on main stems, few on short shoots; corolla lobed for ca. 3/4 of the way to the base; seeds dark brown to almost black; continental Africa north of the equator.

133

132a Calyx lobes fused in fruit, ca. $10 \mathrm{~mm}$ long, fully covering the mature fruit; leaves ovate

Solanum toliaraea D'Arcy \& Rakot.

132b Calyx lobes free in fruit, 4-5 mm long, the mature fruit at least partly exposed; leaves orbicular, elliptic or ovate ........... Solanum batoides D'Arcy \& Rakot.

133a Inflorescences with 1(2) flowers; trichomes on young stem with 12-18 rays.

Solanum cordatum Forssk.

133b Inflorescences with (1)2-20 flowers; trichomes on young stem with 6-10 rays

Solanum forskalii Dunal

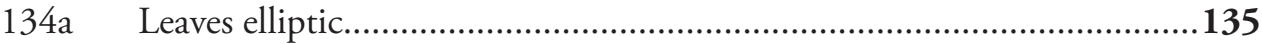

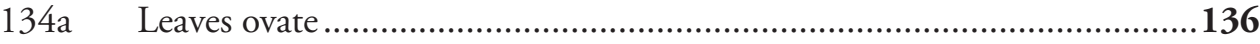

135a Leaves drying yellow-green; anthers 3.5-5.2 mm long; southern Africa

Solanum burchellii Dunal

135b Leaves drying reddish brown; anthers 5.5-8 mm long; Madagascar.

Solanum erythracanthum Dunal

136a Anthers 3.2-3.5 mm long; southern Africa.......... Solanum tomentosum $\mathrm{L}$.

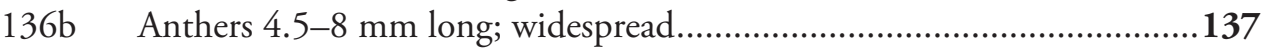

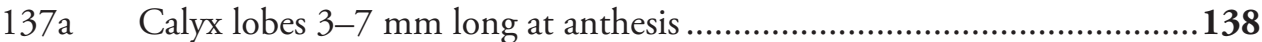

137b Calyx lobes 0.5-3 mm long at anthesis ...............................................139

138a Leaves yellow-green; trichomes on the abaxial sides of the leaves with midpoints 1-2 mm long; Tanzania................... Solanum inaequiradians Bitter

138b Leaves reddish; trichomes on the abaxial sides of the leaves with midpoints to $0.5 \mathrm{~mm}$ long; Madagascar. Solanum myoxotrichum Baker

139a Leaves 6-13 cm long; prickles on young stems 1-2 mm long; highland eastern Africa Solanum stipitatostellatum Dammer

139b Leaves 1-5(6) cm long; prickles on young stems 2-10 mm long; continental Africa, north of the equator or Madagascar..... 140

140a Pedicels 0.2-1 mm long; seeds almost black; continental Africa, north of the equator Solanum forskalii Dunal

140b Pedicels 1.5-2(2.5) cm long; seeds dull yellow to orange-brown; Madagascar Solanum erythracanthum Dunal 


\section{Acknowledgements}

We thank the curators of the herbaria cited in the Appendix 2 for permission to examine specimens in their care; Michael Gilbert for assisting with key reformatting; Sarah Ficinski, Morvah George, Juana de Egea, Samantha Murphy, Xavier Aubriot and David Spooner all greatly helped in the databasing of specimens and in assessing distributions; comments from Leandro Giacomin, Roy Gereau and Gloria E. Barboza greatly improved the manuscript; much of the field and herbarium work was funded by the National Science Foundation Planetary Biodiversity Inventory program grant "PBI Solanum - a worldwide treatment" (DEB-0316614) to SK; other herbarium visits were funded by the SYNTHESYS Project http://www.synthesys.info/ which was financed by European Community Research Infrastructure Actions under the FP6 and FP7 "Structuring the European Research Area" Programme; Natural History Museum Special Funds awards; and the Royal Society through travel grants.

\section{References}

Aubriot X, Singh P, Knapp S (2016) Tropical Asian species show the Old World clade of "spiny solanums" (the Leptostemonum Clade: Solanaceae) is not monophyletic. Botanical Journal of the Linnean Society 180: 1-27. https://doi.org/10.1111/boj.12412

Bohs L (2005) Major clades in Solanum based on $n d h F$ sequences. In: Keating DC, Hollowell VC, Croat TB (Eds) A festschrift for William G. D’Arcy: the legacy of a taxonomist [Monographs in Systematic Botany from the Missouri Botanical Garden, Vol. 104]. Missouri Botanical Garden Press, St. Louis, 27-49.

Bohs L (2007) Phylogeny of the Cyphomandra clade of the genus Solanum (Solanaceae) based on ITS sequence data. Taxon 56(4): 1012-1026. https://doi.org/10.2307/25065901

Bohs L, Olmstead RG (2001) A reassessment of Normania and Triguera (Solanaceae). Plant Systematics and Evolution 228(1-2): 33-48. https://doi.org/10.1007/s006060170035

Brummitt RK (2001) World geographical scheme for recording plant distributions. $2^{\text {nd }}$ edition. Hunt Institute for Botanical Documentation, Pittsburgh. http://web.archive.org/ web/20160125135239/http://www.nhm.ac.uk/hosted_sites/tdwg/TDWG_geo2.pdf [accessed 28 Nov 2018]

Echeverría-Londońo S, Särkinen T, Fenton IS, Knapp S, Purvis A (2018) Dynamism and context dependence in the diversification of the megadiverse genus Solanum L. (Solanaceae). Preprint on bioRxiv. https://doi.org/10.1101/348961

Fawzi NM, Habeeb HR (2016) Taxonomic study of the wild species of Solanum L. in Egypt. Annals of Agricultural Science 61(2): 165-173. https://doi.org/10.1016/j.aoas.2016.10.003

Frodin DG (2004) History and concepts of big plant genera. Taxon 53(3): 753-776. https:// doi.org/10.2307/4135449

Jaeger P-M (1985) Systematic studies in the genus Solanum in Africa. PhD Thesis, University of Birmingham, Birmingham. 
Jaeger P-ML, Hepper FN (1986) A review of the genus Solanum in Africa. In: D'Arcy WG (Ed.) Solanaceae: biology and systematics. Columbia University Press, New York, 41-55. https://doi.org/10.2307/4102956

Knapp S (2013) A revision of the Dulcamaroid clade of Solanum L. (Solanaceae). PhytoKeys 22(0): 1-432. https://doi.org/10.3897/phytokeys.22.4041

Knapp S, Vorontsova MS (2016) A revision of the "African Non-Spiny" Clade of Solanum L. (Solanum sections Afrosolanum Bitter, Benderianum Bitter, Lemurisolanum Bitter, Lyciosolanum Bitter, Macronesiotes Bitter, and Quadrangulare Bitter: Solanaceae). PhytoKeys 66: 1-142. https://doi.org/10.3897/phytokeys.66.8457

Nee M (1979) A revision of Solanum section Acanthophora. PhD dissertation, University of Wisconsin, Madison.

Särkinen T, Olmstead RG, Bohs L, Knapp S (2013) A phylogenetic framework for evolutionary study of the nightshades (Solanaceae): A dated 1000-tip tree. BMC Evolutionary Biology 13(1): 214. https://doi.org/10.1186/1471-2148-13-214

Särkinen T, Barboza GE, Knapp S (2015) True Black nightshades: Phylogeny and delimitation of the Morelloid clade of Solanum. Taxon 64(5): 945-958. https://doi.org/10.12705/645.5

Särkinen T, Poczai P, Barboza GE, van der Weerden GM, Baden M, Knapp S (2018) A revision of the Old World Black Nightshades (Morelloid clade of Solanum L., Solanaceae). PhytoKeys 106: 1-223. https://doi.org/10.3897/phytokeys.106.21991

Stern SR, Agra M de F, Bohs L (2011) Molecular delimitation of clades within New World species of the "spiny solanums" (Solanum subgenus Leptostemonum). Taxon 60(5): 1429_ 1441. https://doi.org/10.1002/tax.605018

Tepe EJ, Anderson GJ, Spooner DM, Bohs L (2016) Relationships among wild realtives of the tomato, porato and pepino. Taxon 65(2): 262-276. https://doi.org/10.12705/652.4

Vorontsova MS, Knapp S (2016) A revision of the "spiny solanums", Solanum subgenus Leptostemonum (Solanaceae) in Africa and Madagascar. Systematic Botany Monographs 99: 1-432.

Weese T, Bohs L (2007) A three-gene phylogeny of the genus Solanum (Solanaceae). Systematic Botany 33(2): 445-463. https://doi.org/10.1600/036364407781179671

Whalen MD (1984) Conspectus of species groups in Solanum subgenus Leptostemonum. Gentes Herbarum 12: 179-282.

Whalen MD, Costich DE, Heiser CB (1981) Taxonomy of Solanum section Lasiocarpa. Gentes Herbarum 12: 41-129. 


\section{Supplementary material I}

\section{List of Solanum species occurring in Africa as defined in this paper}

Authors: Sandra Knapp, Maria S. Vorontsova, Tiina Särkinen

Data type: species data

Copyright notice: This dataset is made available under the Open Database License (http://opendatacommons.org/licenses/odbl/1.0/). The Open Database License $(\mathrm{ODbL})$ is a license agreement intended to allow users to freely share, modify, and use this Dataset while maintaining this same freedom for others, provided that the original source and author(s) are credited.

Link: https://doi.org/10.3897/phytokeys.127.34326.suppl1

\section{Supplementary material 2}

Specimens examined by the authors for the preparation of these keys

Authors: Sandra Knapp, Maria S. Vorontsova, Tiina Särkinen

Data type: species data

Copyright notice: This dataset is made available under the Open Database License (http://opendatacommons.org/licenses/odbl/1.0/). The Open Database License $(\mathrm{ODbL})$ is a license agreement intended to allow users to freely share, modify, and use this Dataset while maintaining this same freedom for others, provided that the original source and author(s) are credited.

Link: https://doi.org/10.3897/phytokeys.127.34326.suppl2 